\title{
Electrogenic sodium bicarbonate cotransporter NBCe1 regulates pancreatic $\beta$ cell function in type 2 diabetes
}

\author{
Matthew R. Brown, ${ }^{1}$ Heather Holmes, ${ }^{1}$ Kuntol Rakshit, ${ }^{1}$ Naureen Javeed, ${ }^{1}$ Tracy K. Her, ${ }^{1}$ Alison A. Stiller, ${ }^{1}$ Satish Sen, ${ }^{1}$ \\ Gary E. Shull, ${ }^{2}$ Y.S. Prakash, ${ }^{1,3}$ Michael F. Romero, ${ }^{1,4}$, and Aleksey V. Matveyenko ${ }^{1,5}$ \\ 'Department of Physiology and Biomedical Engineering, Mayo Clinic College of Medicine and Science, Rochester, Minnesota, USA. 'Department of Molecular Genetics, Biochemistry, and Microbiology, \\ University of Cincinnati College of Medicine, Cincinnati, Ohio, USA. ${ }^{3}$ Department of Anesthesiology, ${ }^{4}$ Division of Nephrology and Hypertension and ${ }^{5}$ Division of Endocrinology, Metabolism, Diabetes, and \\ Nutrition, Department of Medicine, Mayo Clinic College of Medicine and Science, Rochester, Minnesota, USA.
}

\begin{abstract}
Pancreatic $\beta$ cell failure in type 2 diabetes mellitus (T2DM) is attributed to perturbations of the $\beta$ cell's transcriptional landscape resulting in impaired glucose-stimulated insulin secretion. Recent studies identified SLC4A4 (a gene encoding an electrogenic $\mathrm{Na}^{+}$-coupled $\mathrm{HCO}_{3}{ }^{-}$cotransporter and intracellular $\mathrm{pH}$ regulator, $\mathrm{NBCe1}$ ) as one of the misexpressed genes in $\beta$ cells of patients with T2DM. Thus, in the current study, we set out to test the hypothesis that misexpression of SLC4A4/ NBCe1 in T2DM $\beta$ cells contributes to $\beta$ cell dysfunction and impaired glucose homeostasis. To address this hypothesis, we first confirmed induction of SLC4A4/NBCe1 expression in $\beta$ cells of patients with T2DM and demonstrated that its expression was associated with loss of $\beta$ cell transcriptional identity, intracellular alkalinization, and $\beta$ cell dysfunction. In addition, we generated a $\beta$ cell-selective SIc4a4/NBCe1-KO mouse model and found that these mice were protected from diet-induced metabolic stress and $\beta$ cell dysfunction. Importantly, improved glucose tolerance and enhanced $\beta$ cell function in SIc4a4/NBCe1-deficient mice were due to augmented mitochondrial function and increased expression of genes regulating $\beta$ cell identity and function. These results suggest that increased $\beta$ cell expression of SLC4A4/NBCe1 in T2DM plays a contributory role in promotion of $\beta$ cell failure and should be considered as a potential therapeutic target.
\end{abstract}

\section{Introduction}

Pancreatic $\beta$ cells exert tight transcriptional control over key cellular processes (e.g., glucose metabolism, mitochondrial function, ER homeostasis) in order to facilitate the efficient coupling of glucose uptake, oxidation, mitochondrial metabolism, and glucose-stimulated insulin secretion (GSIS; ref. 1). However, the development of type 2 diabetes mellitus (T2DM) is associated with perturbations of the $\beta$ cell's transcriptional landscape, resulting in aberrant regulation of GSIS partly attributed to loss of mitochondrial function, which is a major component of $\beta$ cell failure in T2DM (2-6). Therefore, identifying novel genetic/phenotypic alterations in T2DM $\beta$ cells can greatly enhance our understanding of the pathophysiology.

Recent studies suggest that genetic and phenotypic changes in T2DM $\beta$ cells resemble those of cancer cells, which have been postulated to provide $\beta$ cells a survival advantage at the expense of insulin secretory function (5-8). A hallmark feature of the cancer cell phenotype is a "reverse pH gradient" characterized by acidification of the extracellular environment with concomitant intracellular alkalinization (increased $\mathrm{pH}_{\mathrm{i}}$; ref. 9). Indeed, the alkaline $\mathrm{pH}_{\mathrm{i}}$ of cancer cells is integral in facilitating metabolic adaptions through increased intracellular

Conflict of interest: The authors have declared that no conflict of interest exists. Copyright: () 2021, American Society for Clinical Investigation.

Submitted: July 20, 2020; Accepted: July 22, 2021; Published: September 1, 2021.

Reference information: / Clin Invest. 2021;131(17):e142365.

https://doi.org/10.1172/JCl142365.
$\mathrm{HCO}_{3}{ }^{-}$buffering capacity, permitting reliance of cancer cells on nonoxidative glycolysis and lactate production and leading to diminished mitochondrial function $(9,10)$. In addition, the alkaline $\mathrm{pH}_{\mathrm{i}}$ of cancer cells also contributes to the regulation of enzymatic activity, the epigenetic control of global gene expression, and the promotion of DNA synthesis in response to various growth factors $(9,11,12)$. Consequently, cancer cells maintain an alkaline $\mathrm{pH}_{\mathrm{i}}$ by upregulating expression of ion pumps/transporters that facilitate cellular $\mathrm{H}^{+}$efflux (e.g., $\mathrm{Na}^{+} / \mathrm{H}^{+}$exchanger; $\mathrm{NHE} 1$ ) and $\mathrm{HCO}_{3}^{-}$influx (e.g., $\mathrm{Na}^{+}$-coupled $\mathrm{HCO}_{3}{ }^{-}$cotransporter, $\mathrm{SLC} 4 / \mathrm{NBC}$ family of bicarbonate transporters; refs. 9, 10, 13-16).

Interestingly, a recent single-cell transcriptomic study of the human endocrine pancreas identified SLC4A4 (gene encoding $\mathrm{Na}^{+} / \mathrm{HCO}_{3}{ }^{-}$cotransporter, $\mathrm{NBCe} 1$ ) as one of the few unique genes enriched in immature/dedifferentiated T2DM $\beta$ cells and repressed in nondiabetic (ND) adult $\beta$ cells (17). The SLC4 transporter family encodes 10 human gene products, all of which function to transport $\mathrm{HCO}_{3}{ }^{-}$along with $\mathrm{Na}^{+}$ (or exchange $\mathrm{Cl}^{-}$; ref. 18). Specifically, $\mathrm{NBCe} 1$ is a polytopic membrane protein that primarily functions to mediate unilateral movement of $1 \mathrm{Na}^{+}$ion and $2 \mathrm{HCO}_{3}{ }^{-}$ions across plasma membranes, and the directionality of transport is determined by membrane potential and concentrations of $\mathrm{Na}^{+}$and $\mathrm{HCO}_{3}{ }^{-}(19,20)$. Importantly, NBCe1 plays an integral role in $\mathrm{pH}_{\mathrm{i}}$ regulation, and consequently, increased NBCe1 expression and activity are associated with intracellular alkalinization as commonly occurs in cancer cell lines $(9,10,16,21,22)$, includ- 
A

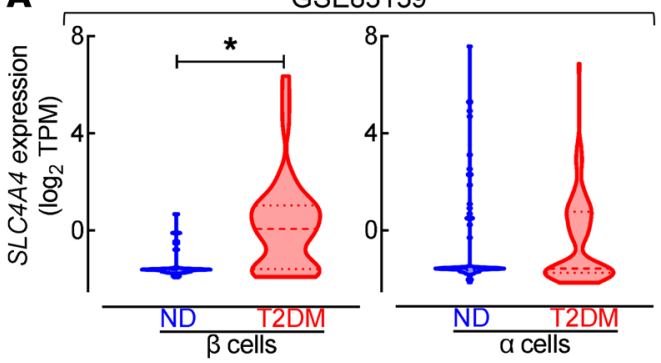

B

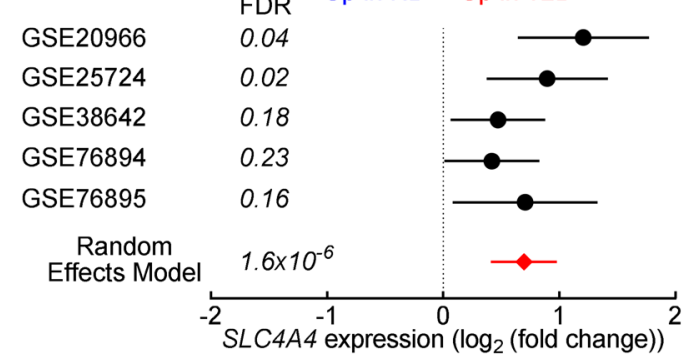

C

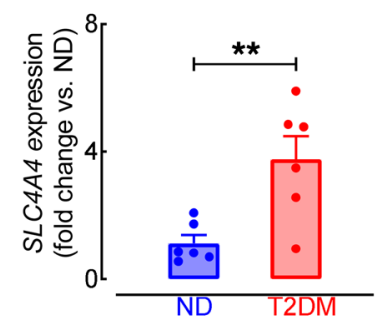

D

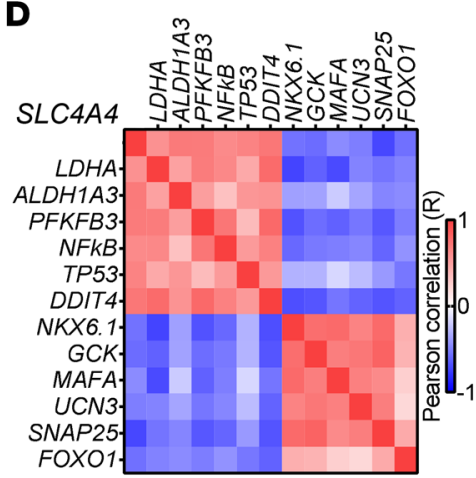

$\mathbf{E}$

KEGG Pathways

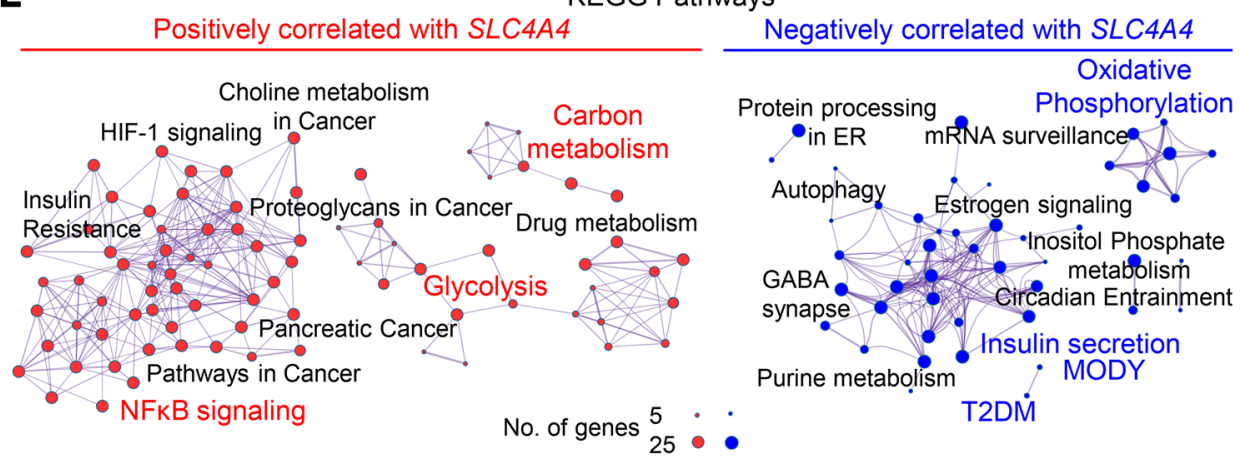

$\mathbf{F}$
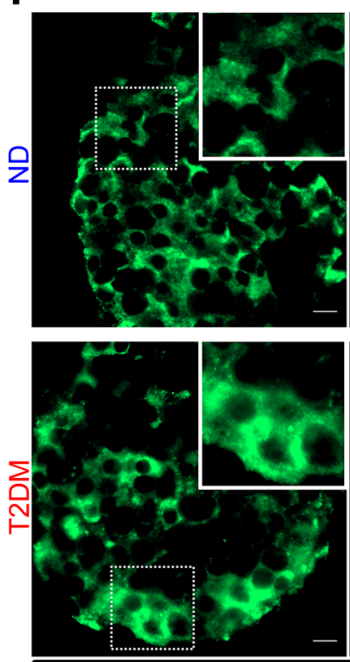
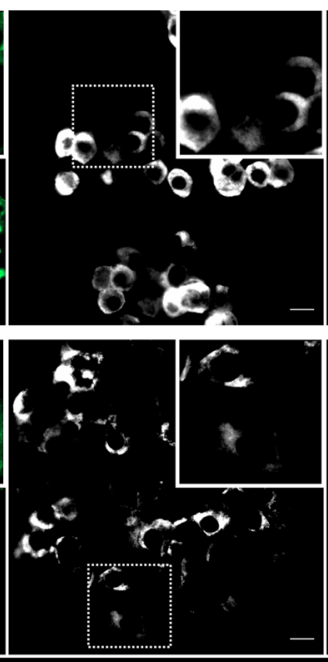
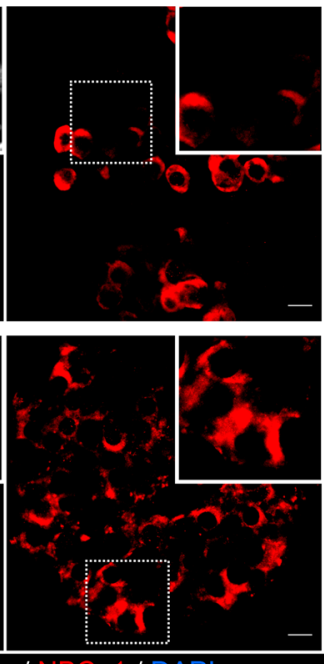
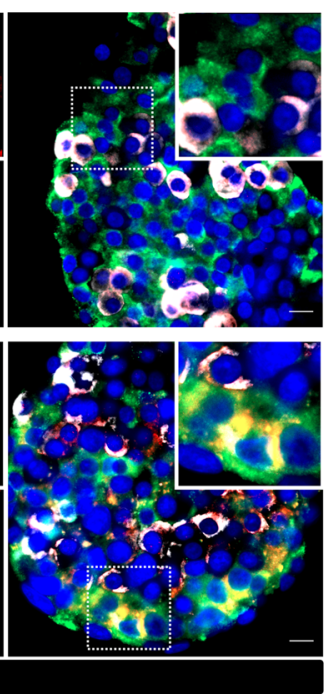

G

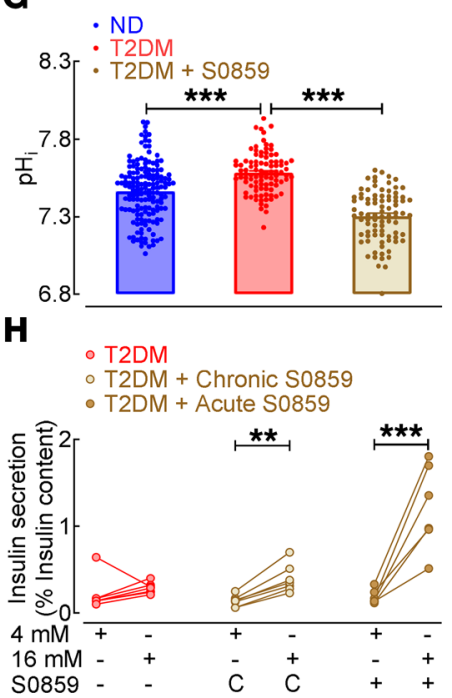

Figure 1. SLC4A4 expression is induced in human islets in type 2 diabetes. (A) SLC4A4 expression in human nondiabetic (ND) and type 2 diabetic (T2DM) $\beta$ and $\alpha$ cells from CSE83139. *FDR < 0.05 (Benjamini-Hochberg method; $n=38-100$ cells). (B) Meta-analysis of SLC4A4 expression in ND and T2DM human islets from 5 whole-gene array studies. FDR < 0.05, denotes statistical significance (Benjamini-Hochberg method). (C) SLC4A4 expression in ND and T2DM isolated human islets. ${ }^{*} P<0.01$ denotes statistical significance (unpaired, 2 -tailed $t$ test; $n=6$ independent ND and T2DM islet shipments). (D) Analysis of CSE38642 using Pearson's correlation of SLC4A4 with key transcripts associated with regulation of $\beta$ cell identity, function, or failure (correlation coefficient; $|\mathrm{R}|=0.5$ to 1 ; FDR $<5 \% ; n=51$ islet samples). (E) Network analysis illustrating the interaction between enriched KEGG pathways ( $P<0.05)$ from genes with statistically significant correlation with SLC4A4. (F) Representative examples of ND and T2DM human islets ( $n=3$ islet shipments/condition) immunostained for NBCe1 (red), insulin (green), and glucagon (white) and imaged at 40x original magnification. Scale bars: $10 \mu \mathrm{m}$. (G) Intracellular pH (pH) in ND, T2DM, and T2DM human islet cells exposed to NBCe1 inhibition (30 $\mu \mathrm{M}$ S0859). ${ }^{* *} P<0.001$ denotes statistical significance (1-way ANOVA with Tukey's method for multiple comparisons; $n=86-158$ independent cells from 2 ND and 3 T2DM islet shipments). (H) Clucose-stimulated insulin secretion (CSIS) at 16 mM and 4 mM glucose in T2DM human islets exposed to (a) vehicle, (b) chronic NBCe1 inhibition (30 $\mu$ M S0859) for 72 hours, or (c) acute NBCe1 inhibition (30 $\mu$ M S0859 in glucose KRBH buffer). Islets exposed to chronic NBCe1 inhibition were not exposed to S0859 during CSIS. ${ }^{* *} P<0.01,{ }^{* *} P<0.001$ denotes statistical significance (unpaired, 2-tailed $t$ test; $n=6-7$ independent experiments from 3 islet shipments). All values are represented as mean \pm SEM.

ing tumoral $\beta$ cells (23). Indeed, previous studies reported the importance of $\mathrm{pH}_{\mathrm{i}}$ for proper regulation of GSIS $(24,25)$; in isolated rodent islets, cytosolic acidification (decreased $\mathrm{pH}_{\mathrm{i}}$ ) was shown to enhance GSIS and alkalinization was shown to repress GSIS (25). Moreover, decreasing islet cell $\mathrm{pH}_{\mathrm{i}}$ with dimethyl amiloride was demonstrated to augment time-dependent potentiation of insulin secretion and improve glucose metabolism in T2DM rodent models $(24,26,27)$. 
Despite increased insights into the regulation of $\beta$ cell transcriptional identity in T2DM, the contribution of $\mathrm{HCO}_{3}^{-}$buffering, $\mathrm{pH}_{\mathrm{i}}$ regulation, and $S L C 4 A 4 / \mathrm{NBCe} 1$ misexpression to $\beta$ cell functional failure in T2DM remains unexplored. Correspondingly, our study provides evidence that induction of SLC4A4/NBCe1 is a notable feature of human $\beta$ cells in T2DM, where its expression promotes $\mathrm{pH}_{\mathrm{i}}$ alkalinization and impaired mitochondrial function and contributes to $\beta$ cell functional failure and glucose intolerance.

\section{Results}

SLC4A4 expression is induced in human islets in T2DM and its expression is associated with $\beta$ cell dysfunction. Wang et al. (17) through single-cell RNA-Seq of the human endocrine pancreas identified SLC4A4 (gene encoding $\mathrm{Na}^{+}$-coupled $\mathrm{HCO}_{3}{ }^{-}$cotransporter NBCe1) as one of the unique genes enriched in immature (child) and adult T2DM $\beta$ cells (Figure 1A; FDR < 0.05 vs. ND; NCBI's Gene Expression Omnibus [GEO] GSE83139; ref. 17). We confirmed the induction of SLC4A4 expression in T2DM human islets by performing a meta-analysis of 5 independent cohorts that examined differential gene expression in human ND vs. T2DM islets (GEO GSE38642; ref. 28, GEO GSE25724; ref. 29, GEO GSE20966; ref. 30, GEO GSE76894; ref. 31, GEO GSE76895; ref. 31 and Figure 1B; FDR < 0.05 vs. ND). To further confirm these observations, we independently procured ND and T2DM human islets (Supplemental Table 1; supplemental material available online with this article; https://doi.org/10.1172/JCI142365DS1) and observed an approximately 3-fold increase in SLC4A4 mRNA in T2DM islets (Figure 1C; $P<0.01$ vs. ND). Meanwhile, mRNA levels of SLC9A1 (NHE1), the primary $\mathrm{Na}^{+} / \mathrm{H}^{+}$exchanger and another key regulator of $\mathrm{pH}_{\mathrm{i}}$, were unchanged in ND versus T2DM $\beta$ cells and whole islets (Supplemental Figure 1, A-C; $P>0.05$ ND vs. T2DM). We next examined how the islet's transcriptional identity changes in concert with rising SLC4A4 mRNA levels (Figure $1, \mathrm{D}$ and $\mathrm{E})$. Indeed, in a large data set with 51 islet donors with known HbA1c (GEO GSE38642; ref. 28), SLC4A4 mRNA showed a robust positive correlation (Pearson correlation $|\mathrm{R}|>0.5$; FDR $<$ 0.05) with the expression of transcripts (e.g., ALDH1A3, PFKFB3, LDHA) collectively annotated to Kyoto Encyclopedia of Genes and Genomes (KEGG) biological pathways associated with $\beta$ cell failure (e.g., HIF1 $\alpha$ signaling, NF- $\kappa \mathrm{B}$ signaling, glycolysis). In contrast, SLC4A4 expression negatively correlated with transcripts (e.g., NKX6.1, MAFA, UCN3) and pathways regulating $\beta$ cell secretory function and identity (e.g., insulin secretion, oxidative phosphorylation; Figure 1E).

Since SLC4A4 encodes an electrogenic $\mathrm{Na}^{+}-\mathrm{HCO}_{3}^{-}$cotransporter (NBCe1) critical for $\mathrm{pH}_{\mathrm{i}}$ regulation (18), we next assessed whether increased SLC4A4 mRNA affects $\mathrm{pH}_{\mathrm{i}}$ and GSIS in human T2DM islets. First, we confirmed increased $\beta$ cell immunoreactivity for NBCe1 in isolated human islets of patients with T2DM (Figure $1 F)$. Next, we measured $\mathrm{pH}_{\mathrm{i}}$ using carboxy-seminaphthorhodofluor-5 (SNARF5), a ratiometric/fluorescent $\mathrm{pH}$ sensor $(24,32)$. T2DM islet cells exhibited significantly higher $\mathrm{pH}_{\mathrm{i}}(+0.1 \mathrm{pH}$ unit vs. ND cells), mirroring $\mathrm{pH}_{\mathrm{i}}$ changes reported in noncancerous versus cancer cells (Figure 1G; $P<0.001$ vs. ND cells). Notably, treatment of T2DM islets with a known NBCe1 inhibitor, S0859 (31), resulted in intracellular acidification (Figure $1 G ; P<0.0001$ vs. T2DM cells) and corresponding restoration of GSIS after either chronic or acute treatment with the NBCe1 inhibitor (Figure 1H; $P<0.001,16 \mathrm{mM}$ vs. $4 \mathrm{mM}$ glucose). In addition, the reduction of $\mathrm{pH}_{\mathrm{i}}$ with a permeable weak acid (butyrate) also augmented GSIS response in T2DM islets, confirming intracellular acidification as a strategy to enhance GSIS in T2DM (Supplemental Figure 2). Importantly, S0859 treatment did not affect GSIS in ND islets (Supplemental Figure 3). Taken together, these data suggest that SLC4A4 expression is induced in human islets in T2DM where its expression and activity are associated with loss of $\beta$ cell transcriptional identity, intracellular alkalinization, and $\beta$ cell dysfunction.

Obesity and T2DM are characterized by marked expression of the $\mathrm{Na}^{+}-\mathrm{HCO}_{3}^{-}$cotransporter, $\mathrm{NBCe1}$, in human $\beta$ cells. To extend our findings, we next used immunofluorescence coimmunostaining of human pancreatic autopsy specimens to assess NBCe1 expression patterns in $\beta$ (and $\alpha$ ) cells of lean ND $\left(\mathrm{BMI}<29.5 \mathrm{~kg} / \mathrm{m}^{2}\right)$, obese $\mathrm{ND}\left(\mathrm{BMI} \geq 29.5 \mathrm{~kg} / \mathrm{m}^{2}\right)$, and obese T2DM $\left(\mathrm{BMI} \geq 29.5 \mathrm{~kg} / \mathrm{m}^{2}\right)$ agematched individuals (Supplemental Table 2). To be included in the analysis, pancreatic tissue must have been obtained within 6 to 8 hours of death to avoid confounding effects of tissue autolysis and linked to a comprehensive, longitudinal medical record that included documented diabetes status. Consistent with gene expression data, we observed minimal NBCe1 immunoreactivity in $\beta$ cells of lean ND individuals but demonstrated robust expression in $\beta$ cells from obese ND and obese T2DM individuals (Figure $2 \mathrm{~A}$ ). Objectively, we found a significant increase in insulin ${ }^{+}$, $\mathrm{NBCe}^{+}$immunoreactivity in obese T2DM individuals (Figure 2B; $P<0.01$ vs. lean ND). There was no significant change in NBCe1 expression in glucagon-positive $\alpha$ cells between lean ND and obese T2DM patients (Figure 2B; $P>0.05$ vs. lean ND). Moreover, expression of NHE1, a key $\mathrm{pH}_{\mathrm{i}}$ regulator (encoded by SLC9A1), was unchanged in T2DM $\beta$ or $\alpha$ cells compared with ND (Supplemental Figure 1D). Given our previous observation that $S L C 4 A 4$ mRNA was increased in parallel with loss of key genes regulating $\beta$ cell identity (e.g., NKX6.1, MAFA, and UCN3), we sought to identify whether these changes were mirrored at the protein level in human $\beta$ cells. We observed that $\beta$ cell NBCe1 induction occurred in conjunction with the nuclear to cytoplasmic redistribution of NKX6.1 in T2DM $\beta$ cells (Figure 2C), a marker of $\beta$ cell failure and loss of transcriptional identity in T2DM (33). Consistent with previous work in human pancreatic autopsy specimens (34), we observed that $67 \%$ of T2DM $\beta$ cells had cytoplasmic NKX6.1 and NBCe1 immunoreactivity (Figure 2D; $P<0.001$ vs. ND), while $60 \%$ of ND $\beta$ cells had negligible NBCe1 and nuclear NKX6.1 immunoreactivity (Figure 2D; $P<0.001$ vs. T2DM).

Diabetogenic stressors induce SLC4A4 expression and increase $\mathrm{pH}_{i}$ in mouse and human islets. Next, we set out to identify which cellular stressors commonly associated with $\beta$ cell failure in T2DM contribute to the induction of $S L C 4 A 4 / \mathrm{NBCe} 1$ in $\beta$ cells (Figure 3). Existing literature shows that the $S L C 4 A 4$ promoter contains $\mathrm{pH}$-dependent domains for several transcription factors commonly associated with $\beta$ cell failure in T2DM, such as stress-associated jun-proto-oncogene (cJUN), proinflammatory NF- $\mathrm{B}$, DNA damage-associated tumor protein p53 (TP53), and TGF- $\beta$ signaling-induced Smad4 $(12,13)$. We extended these findings by performing unbiased network analysis illustrating the interaction between 69 potential transcription factors predicted to bind to the promoter of SLC4A4 by the ENCODE project (Figure 3A). Consistently, 

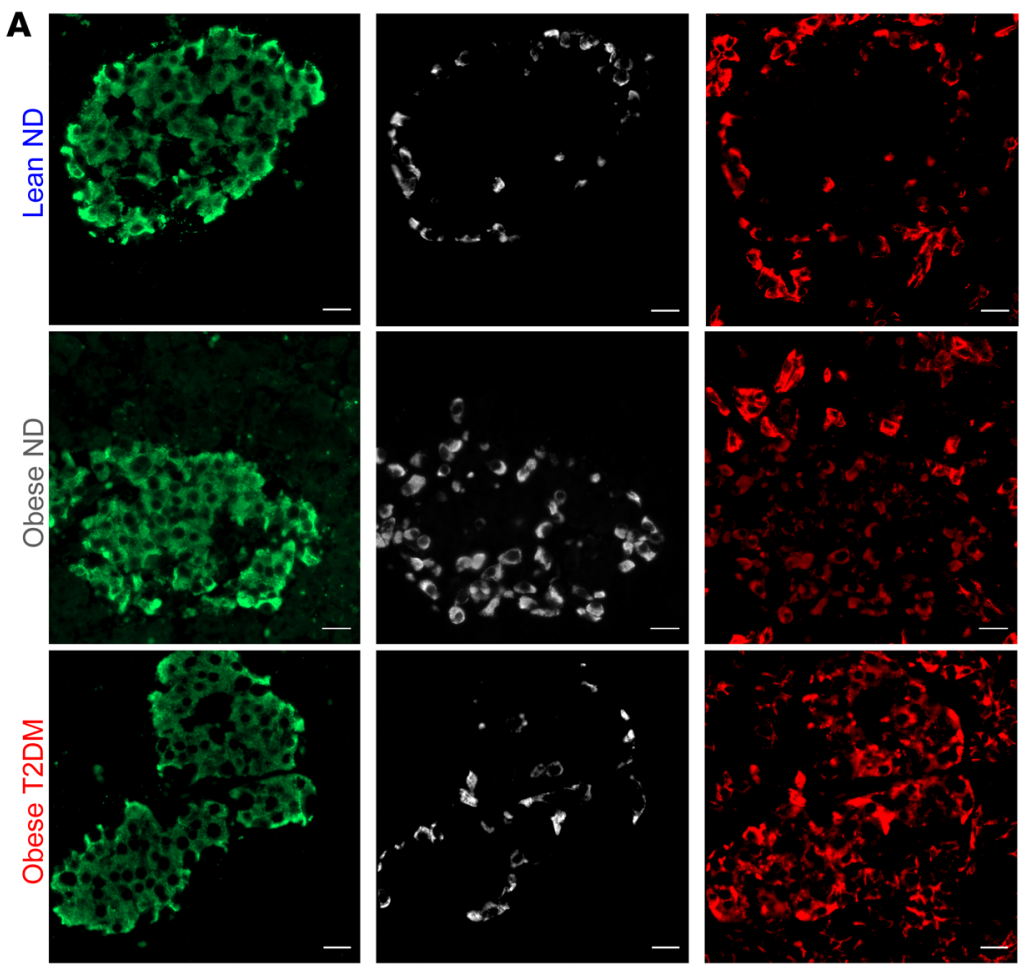

C
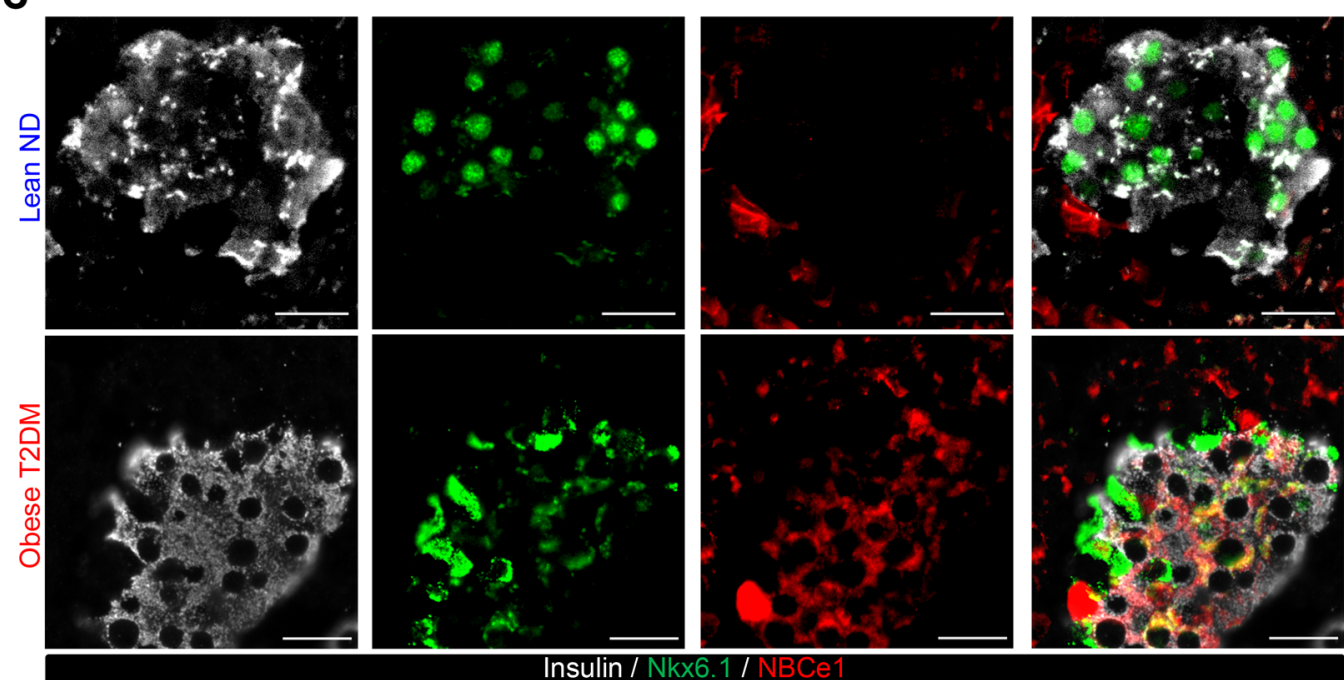
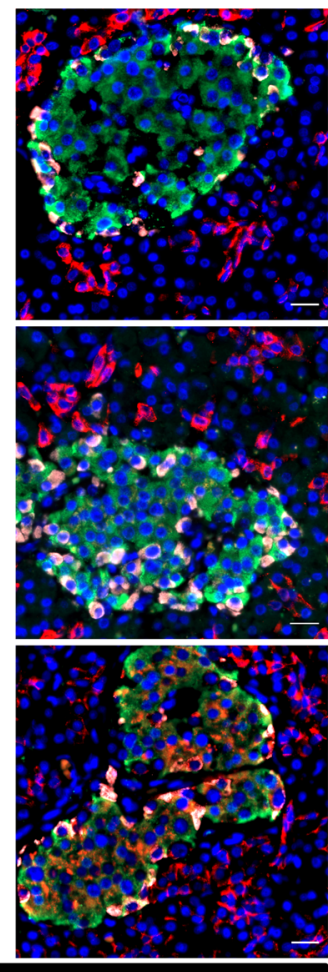

B
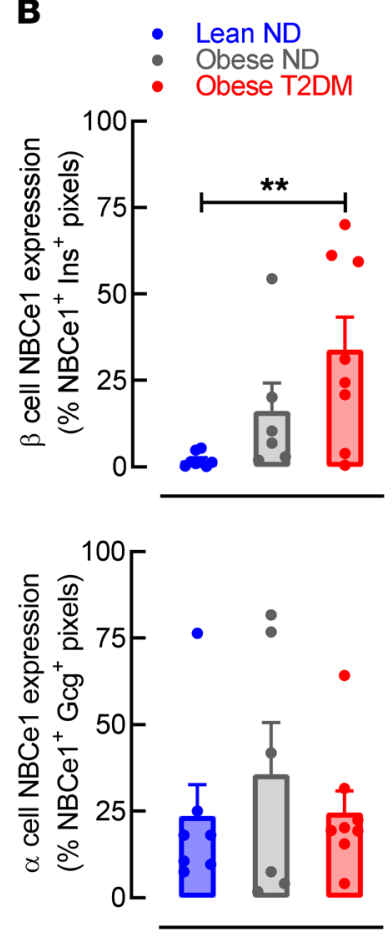

D

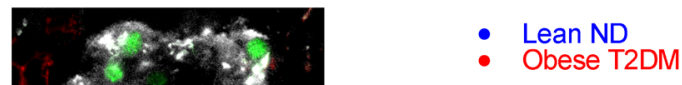

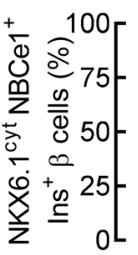

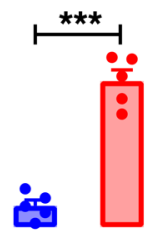

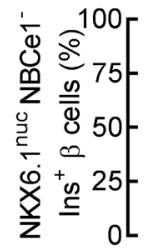

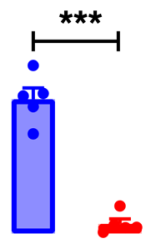

Figure 2. NBCe1 expression is increased in T2DM $\beta$ cells and is associated with loss of $\boldsymbol{\beta}$ cell identity. (A) Representative examples of human pancreatic sections immunostained for NBCe1 (red), insulin (green), glucagon (white), and nuclear marker DAPI (blue) and imaged at 20x magnification. Human pancreas was obtained at autopsy from lean, nondiabetic (lean ND); obese nondiabetic individuals (obese ND); and patients with documented obesity and T2DM (obese T2DM). Scale bars: $20 \mu \mathrm{m}$ in all images. Images are representative of $n=6-8$ independent autopsy specimens per group. (B) Mean NBCe1 expression in $\beta$ and $\alpha$ cells from lean ND, obese ND, and obese T2DM individuals as represented by the percentage of NBCe1 and insulin (top) or glucagon (bottom) double-positive pixels. ${ }^{*} P<0.01$ denotes statistical significance versus lean ND (1-way ANOVA with Dunnett's method for multiple comparisons; $n=6-8$ independent autopsy specimens per group). (C) Representative images of human pancreatic sections from lean ND and obese T2DM individuals immunostained for NBCe1 (red), insulin (gray), and NKX6.1 (green) and imaged at 63× magnification. Scale bars: $20 \mu \mathrm{m}$ in all images. Images are representative of $n=5$ independent autopsy specimens per group. (D) Fraction of $\beta$ cells from lean ND and obese T2DM individuals expressing cytoplasmic NKX6.1 and NBCe1 (top) and fraction of $\beta$ cells expressing nuclear NKX6.1 in the absence of NBCe1 relative to background (bottom). ${ }^{* * *} P<0.001$ denotes statistical significance vs. lean ND (unpaired, 2 -tailed $t$ test; $n=5$ independent autopsy specimens per group). All values are represented as mean \pm SEM.

pathway analysis of the transcription factors predicted to regulate $S L C 4 A 4$ expression revealed enrichment $(P<0.05)$ for pathways associated with $\beta$ cell stress and functional failure (e.g., apoptosis, TGF- $\beta$ signaling, ER stress, inflammation; Figure $3 \mathrm{~A})$. We subse- quently used a mouse $\beta$ cell line (MIN6) to screen common $\beta$ cell stressors as potential mediators of Slc4a4 induction in $\beta$ cells (Figure 3B). Our results showed that the exposure of MIN6 cells to either the pharmacological ER stress inducer thapsigargin and/or 
A Predicted TF Binding to SLC4A4 Promoter

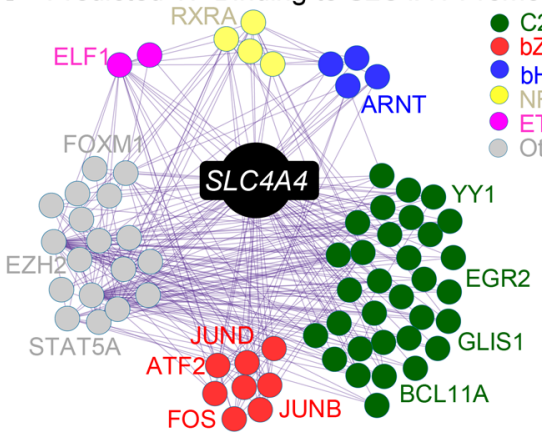

Mouse Islets

C
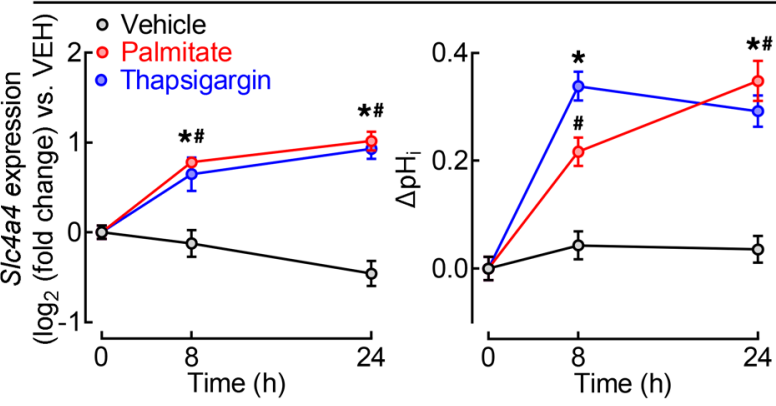

0.0
Enriched Panther Biological Pathways

JAK/STAT signaling pathway

Vitamin D signaling pathway

p53 pathway

Huntington disease

PDGF signaling pathway

Interleukin signaling pathway

CCKR signaling map

TGF $\beta$ signaling pathway

GRH receptor pathway

Apoptosis signaling pathway

G
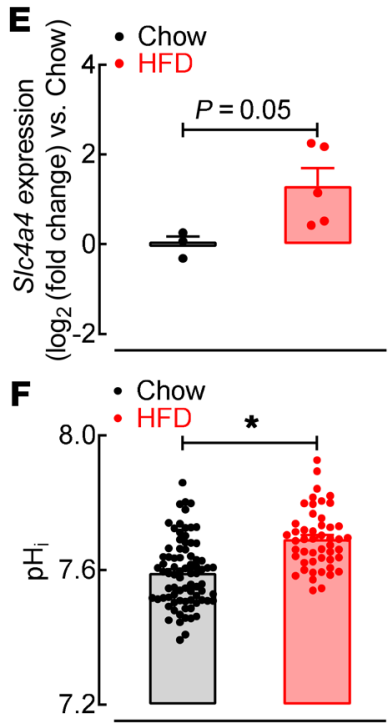
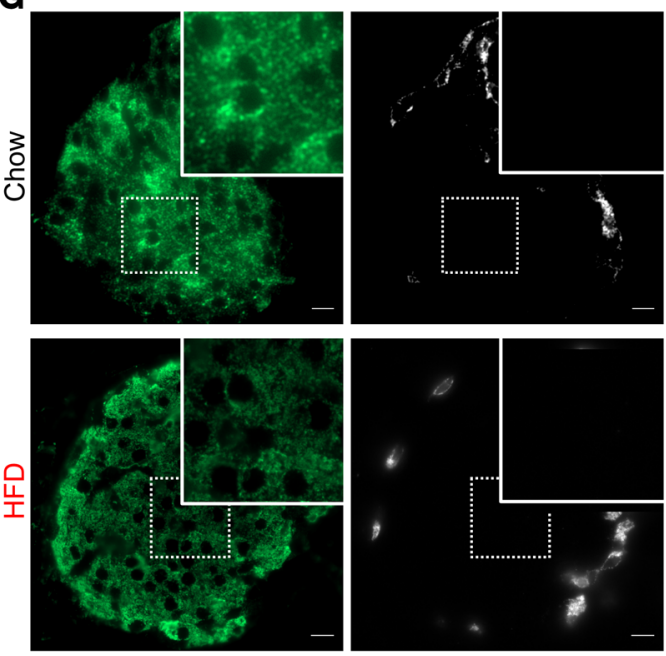

D
B

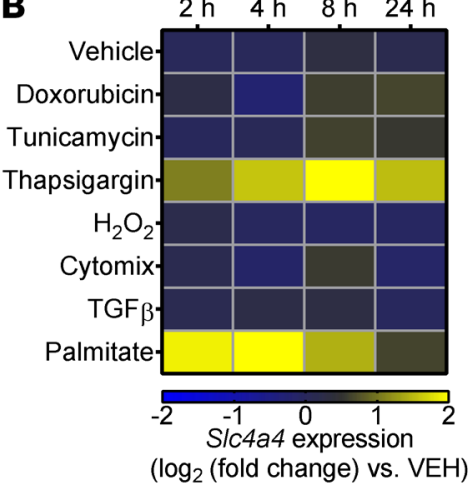
Human Islets
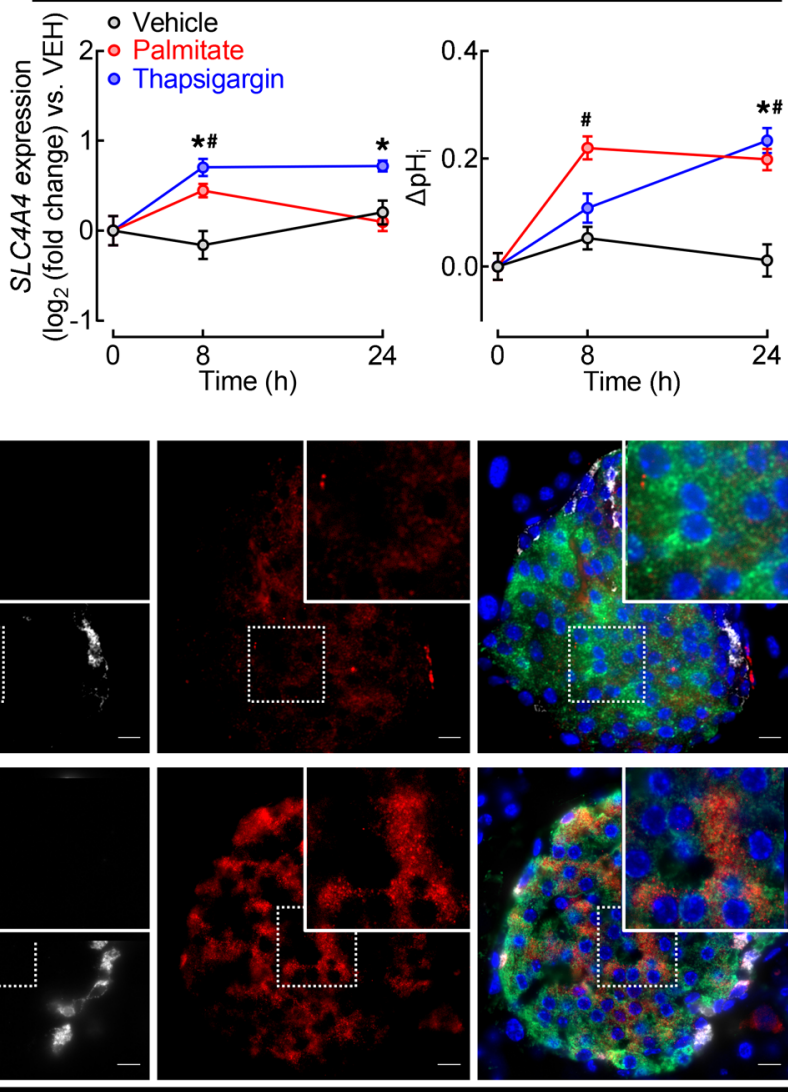

Figure 3. Diabetogenic stressors induce SIc4a4 expression and increase $\mathrm{pH}_{\mathrm{i}}$ in mouse and human islets. (A) Network analysis illustrating interaction between 69 transcription factors predicted to bind to promoter of SLC4A4 (ENCODE project). Key transcription factors associated with $\beta$ cell stress are highlighted (left). Bar graph highlighting top 10 enriched biological pathways from transcription factors predicted to bind to promoter of SLC4A4 (right). (B) Heatmap visualization of SIc4a4 expression in MIN6 cells in response to 2, 4, 8, and 24 hours of diabetogenic stressors: vehicle (DMSO), $200 \mathrm{nM}$ doxorubicin, $1 \mu \mathrm{M}$ tunicamycin, $1 \mu \mathrm{M}$ thapsigargin, $250 \mu \mathrm{M} \mathrm{H}_{2} \mathrm{O}_{2}$, Cytomix ( $0.2 \mathrm{ng} / \mathrm{mL}$ IL- $1 \beta, 10 \mathrm{ng} / \mathrm{mL}$ TNF- $\alpha, 10 \mathrm{ng} / \mathrm{mL}$ IFN- $\left.\gamma\right), 4 \mathrm{ng} / \mathrm{mL}$ TGF- $\beta$, or $0.5 \mathrm{mM}$ palmitate. Data normalized for vehicle with $\beta$-actin as control ( $n=3-4$ independent experiments per treatment/time point). (C and D) S/c4a4 expression (left) and corresponding pH (right) in (C) C57B6J isolated mouse islets or (D) ND human islets in response to $1 \mu \mathrm{M}$ thapsigargin, $0.5 \mathrm{mM}$ palmitate, or vehicle for 8 and 24 hours. ${ }^{*} \#<0.05$ denotes statistical significance versus vehicle (2-way ANOVA with Dunnett's method for multiple comparisons; $n=3$ independent experiments per condition for S/c4a4 mRNA and $n=$ 27-62 independent islet cells per condition for $\mathrm{pHi}$ ). (E) S/c4a4 expression in isolated C57B6) mouse islets exposed to control or 60\% HFD for 8-10 weeks (unpaired, 2-tailed $t$ test, $n=3-5$ mice per group) (86). (F) $\mathrm{pH}_{\mathrm{i}}$ in isolated mouse islets exposed to control or 60\% HFD for 8 to 10 weeks. ${ }^{*} P<0.05$ denotes statistical significance (unpaired, 2-tailed $t$ test, $n=49-79$ independent islet cells per group). (C) Representative images of pancreatic sections and individual islets immunostained for NBCe1 (red), insulin (green), and glucagon (white) and imaged at 40x original magnification from C57B6) mice exposed to control or $60 \%$ HFD for 8 to 10 weeks. Scale bars: $10 \mu \mathrm{m}$. Images representative of $n=3$ mice per group.

saturated fatty acid palmitate resulted in robust induction in Slc4a4 mRNA ( 3-fold vs. vehicle, $P<0.05$; Figure $3 \mathrm{~B}$ ). We followed up on these studies to demonstrate that exposure of primary isolated mouse and human islets to either thapsigargin or palmitate resulted in the increase in Slc4a4 mRNA and corresponding intracellular alkalinization $(P<0.05$ vs. vehicle; Figure 3, C and D). Consistent 
A

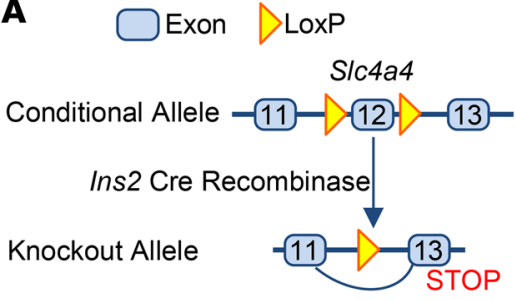

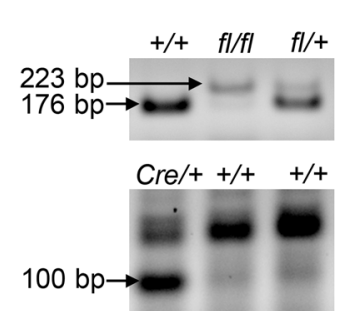

B

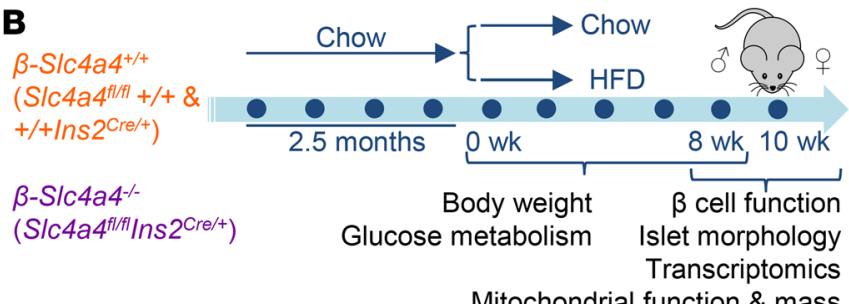

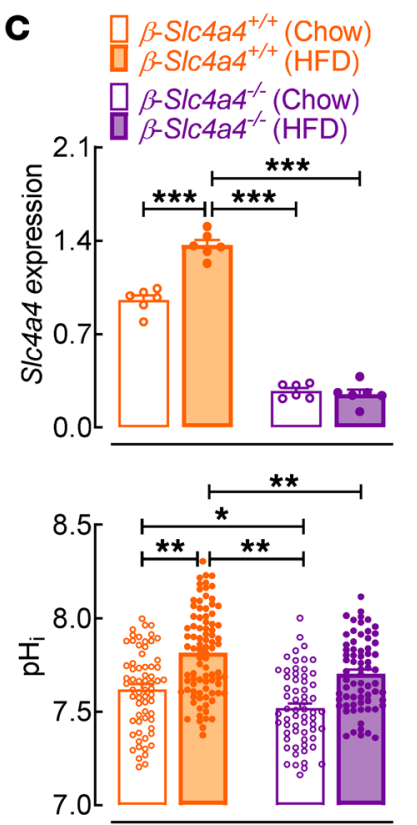

E

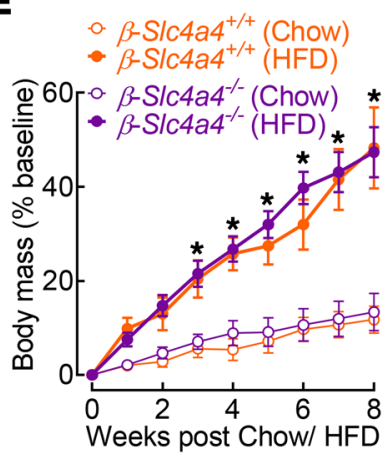

D
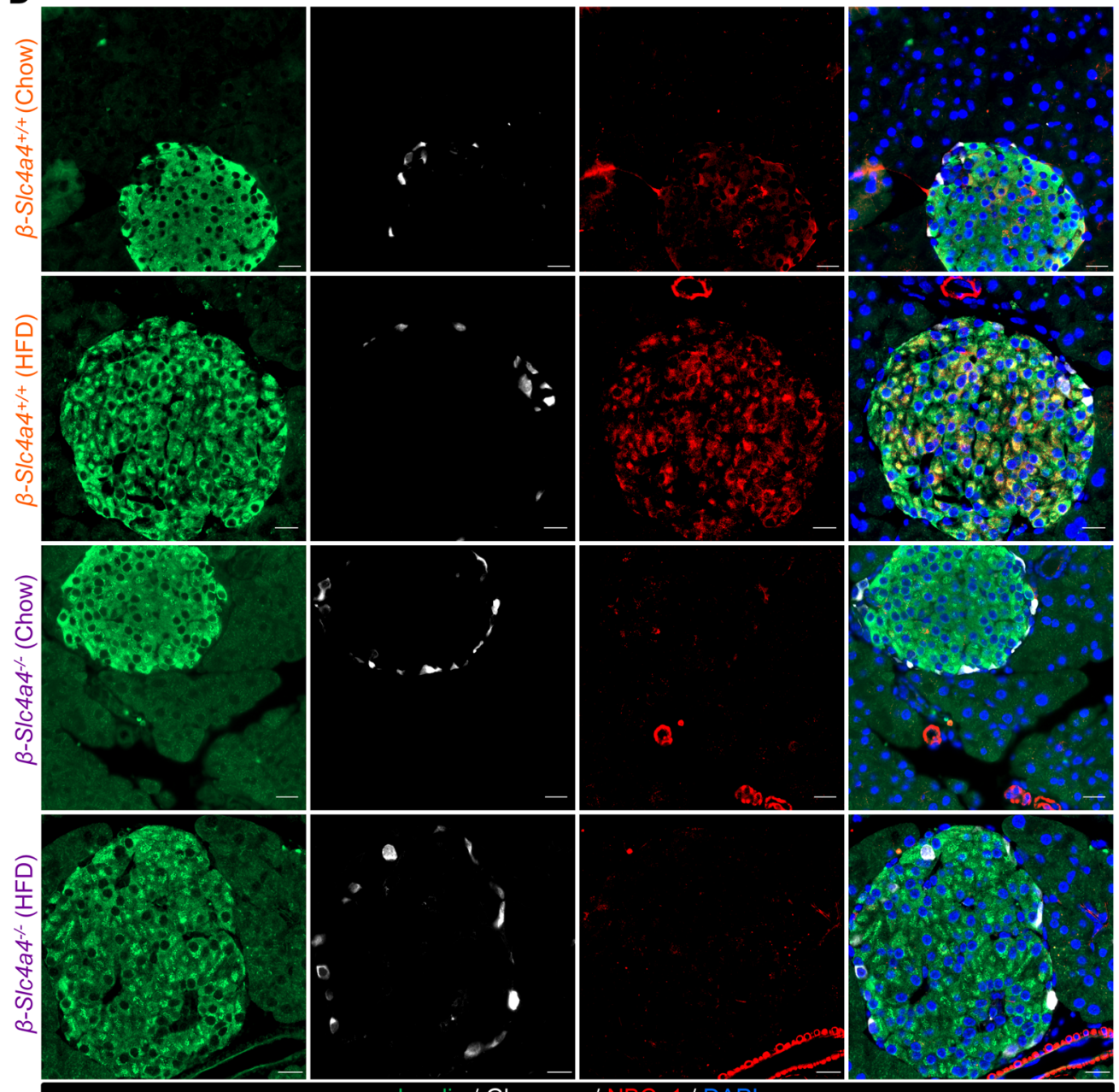

Figure 4. Generation and validation of a $\boldsymbol{\beta}$ cell-selective SIc4a4-null mouse model. (A) Targeting strategy for $\beta$ cell-selective deletion of S/c4a4 (left). Representative PCR analyses of tail DNA using primers for identification of Slc4a4 floxed allele and Cre recombinase (right). (B) Diagram

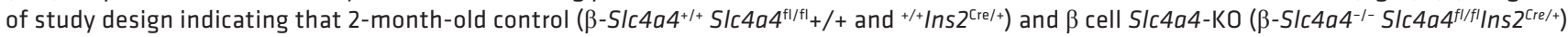
mice were treated for 8 weeks with either chow diet or 60\% HFD. In vivo characterization of glucose metabolism occurred at 0 , 4 , and 8 weeks. Islets were isolated from the mice for functional and transcriptomic profiling; pancreata were isolated for morphological assessment. (c) Slc4a4 expression from $\beta$-SIc $4 a 4^{+/+}\left(S / c 4 a 4^{f l / f l}+/+\right)$ and $\beta$-SIc $4 a 4^{-/-}\left(S / c 4 a 4^{f l / f l} I n s 2^{\text {cre } /+}\right)$ pancreatic islets exposed to either 8 weeks of chow or HFD. ${ }^{* * *} P<$ 0.001 denotes statistical significance versus $\beta$-SIc4a4 ${ }^{+/+}$chow (unpaired, 2-tailed $t$ test; $n=6$ mice per genotype and diet) (top). $\mathrm{pH} \mathrm{H}_{\mathrm{i}}$ measured in

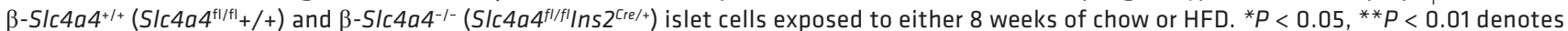
statistical significance (1-way ANOVA with Tukey's method for multiple comparisons; $n=62-87$ independent cells from $n=2$ mice per genotype and diet) (bottom). (D) Representative examples of pancreatic sections and individual islets immunostained for insulin (green), glucagon (white),

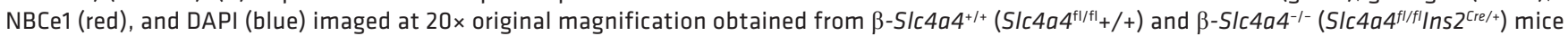
exposed to 8 weeks of chow or HFD. Scale bars: $20 \mu \mathrm{m}$. Images are representative of $n=3$ mice per genotype and diet. (E) Percentage change in

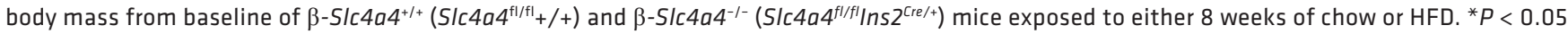
denotes statistical significance versus $\beta$-SIc $4 a 4^{+/+}$chow (2-way ANOVA with Dunnett's method for multiple comparisons; $n=5-7$ mice per genotype and diet). All values are represented as mean \pm SEM. 
A
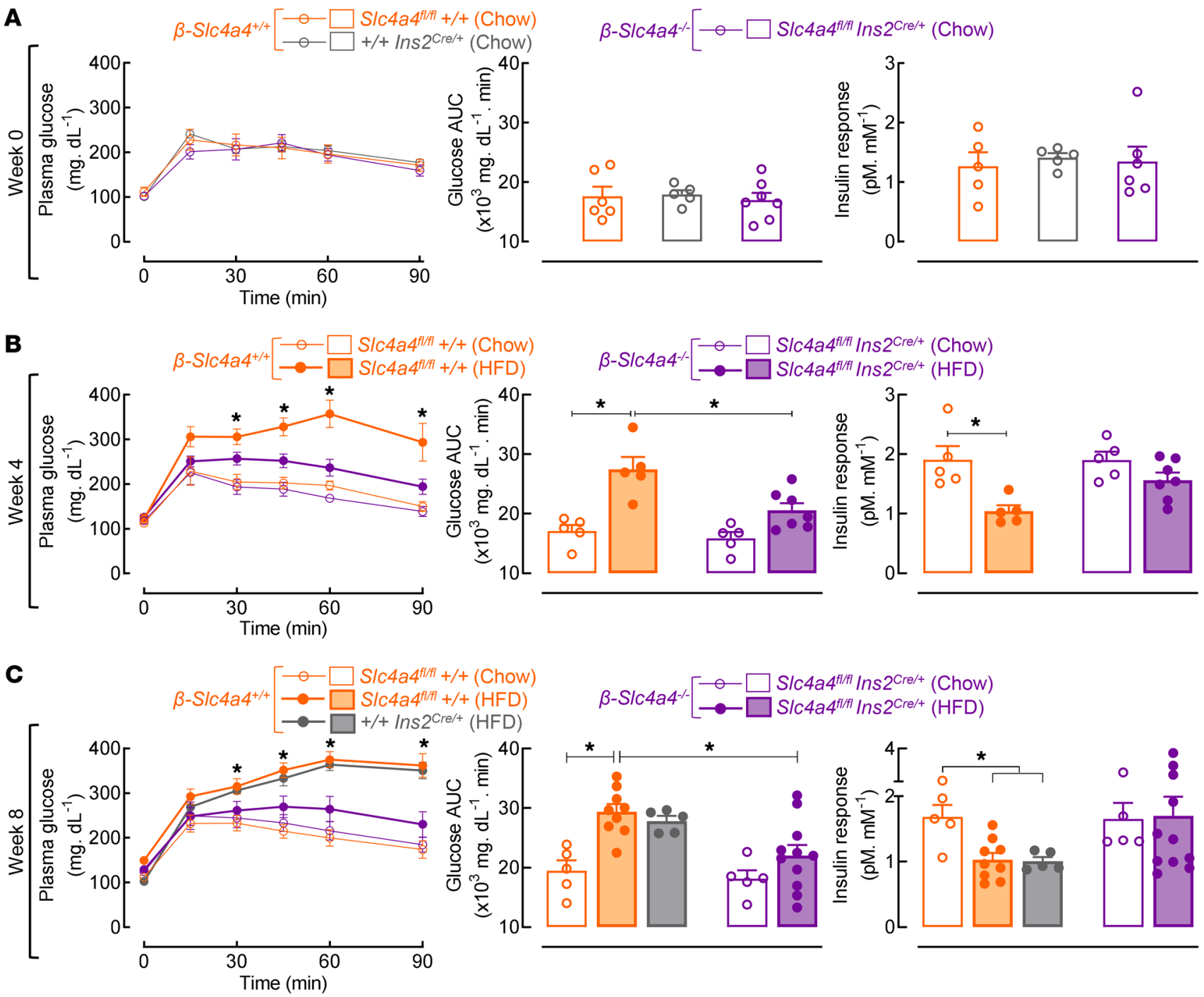

Figure 5. Selective deletion of SIc4a4 in $\beta$ cells protects against high-fat diet-induced glucose intolerance and $\beta$ cell dysfunction in mice in vivo. (A) Glucose tolerance (left), glucose tolerance AUC (center), and corresponding insulin response expressed as the plasma insulin AUC normalized to basal insulin

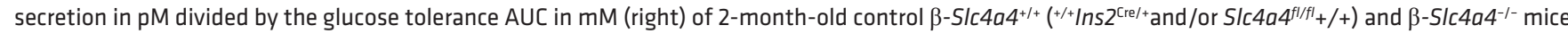

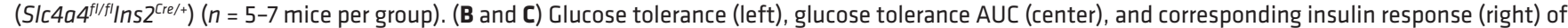

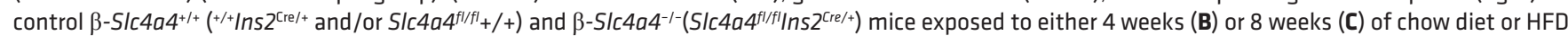
( $n=5$-11 mice per group). For glucose tolerance, ${ }^{*} P<0.05$ denotes statistical significance versus $\beta$-SIc $4 a 4^{+/+}$chow (2-way ANOVA with Dunnett's method for multiple comparisons). For glucose tolerance AUC and insulin response, ${ }^{*} P<0.05$ denotes statistical significance (1-way ANOVA with Dunnett's method for multiple comparisons) as indicated in the text. All values are represented as mean \pm SEM.

with these observations, we also demonstrated that exposure to lipotoxicity in vivo (e.g., 60\% HFD) led to the induction of Slc4a4/ NBCe1 expression and increased islet $\mathrm{pH}_{\mathrm{i}}(P<0.05$ vs. control lowfat diet, Figure 3, E-G). Cumulatively, these studies suggested that the induction of $\beta$ cell Slc4a4 and consequent cytosolic alkalinization was driven by exposure to lipotoxicity/ER stress.

Generation and validation of a $\beta$ cell-selective Slc4a4-null mouse model. To elucidate the functional role of Slc4a4/NBCe1 in $\beta$ cells in response to lipotoxicity-driven obesity and T2DM, we utilized a previously validated $\operatorname{Slc} 4 a 4^{\mathrm{A} / \mathrm{l}}$ mouse with LoxP sites targeting exon 12 of Slc4a4 $(35,36)$ (Figure 4A). The wild-type and floxed alleles were identified by a 178 bp and 223 bp product, respectively, via PCR analysis (Figure. 4A). We subsequently generated $\beta$ cell-selective Slc4a4-KO $\left(\beta-S l c 4 a 4^{-/}\right)$mice through crossing of $S l c 4 a 4^{\mathrm{A} / \mathrm{l}}$ mice with rat insulin
2 promoter-Cre (RIP-Cre) mice (Figure $4 \mathrm{~A}) . \beta-S l c 4 a 4^{-1-}\left(\right.$ Slc $4 a 4^{\text {Af/l }}$ Ins $2^{\mathrm{Cre} /+}$ ) and corresponding $\beta-S l c 4 a 4^{+/+}$controls $\left(S l c 4 a 4^{\mathrm{B} / \mathrm{fl}+}+/+\right.$ and ${ }^{+/}$Ins $2^{\text {Cre/+}}+$ ) were subjected to 8 weeks of either a standard chow diet or a diabetogenic $60 \%$ HFD to model obesity-induced glucose intolerance and $\beta$ cell dysfunction (Figure 4B). In line with our previous observations, expression of both Slc4a4 and NBCe1 was robustly increased in HFD-fed mice (Figure 4, C and D; $P<0.05$ vs. $\beta-S l c 4 a 4^{+/+}$chow). Importantly, we found no detectable NBCe1 expression in $\beta$-Slc4a4 ${ }^{-/-}$ chow and/or HFD $\beta$ cells as well as an approximately $80 \%$ reduction in islet Slc4a 4 mRNA (Figure 4, C and D; $P<0.05$ vs. $\beta-S l c 4 a 4^{+/+}$chow), thus confirming the validity of the generated $\beta-S l c 4 a 4^{-/}$mouse model.

Importantly, to account for potential recombination in the hypothalamus and the expression of passenger human growth hormone cDNA in RIP-Cre mice $(37,38)$, we (a) confirmed lack 

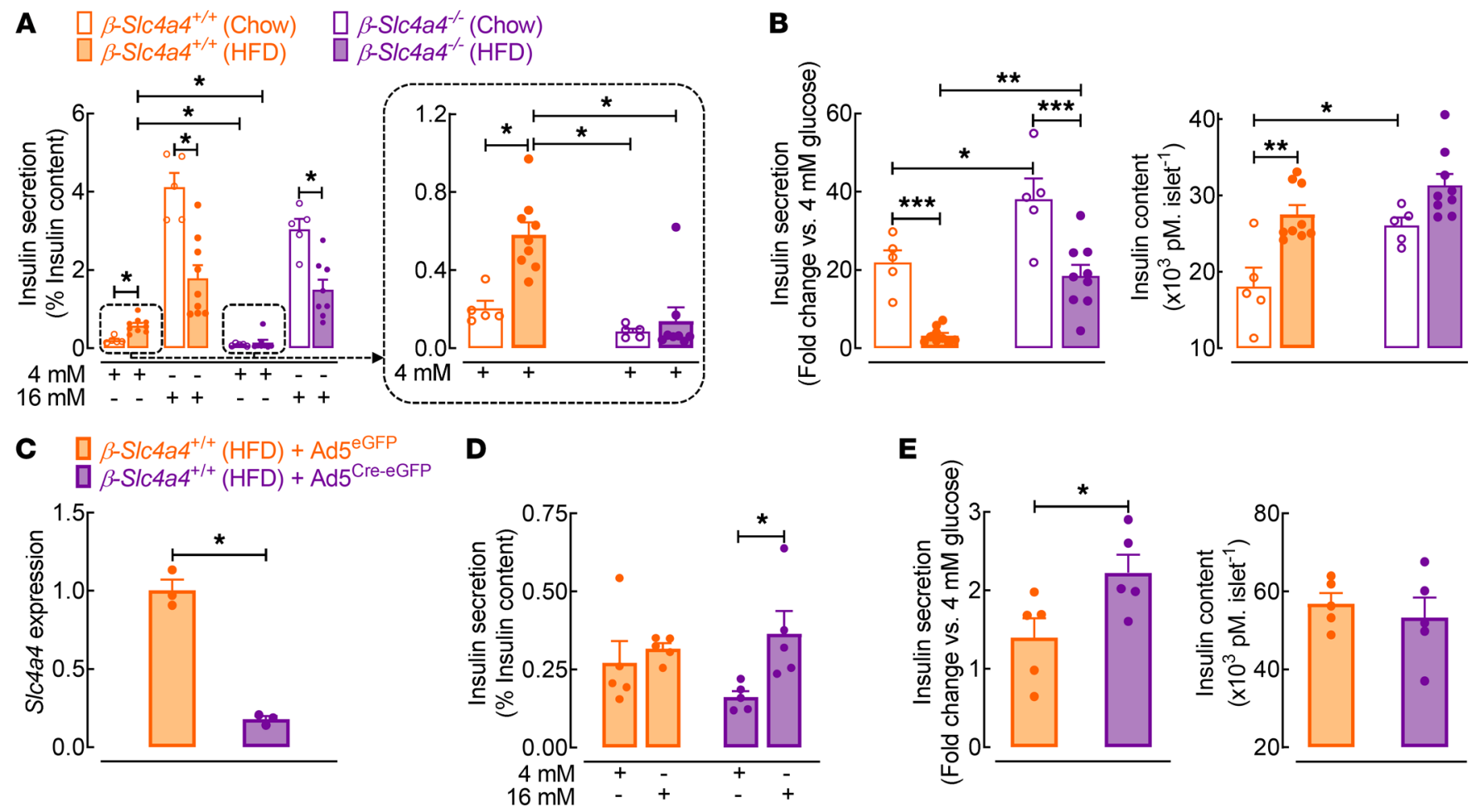

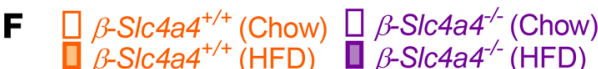
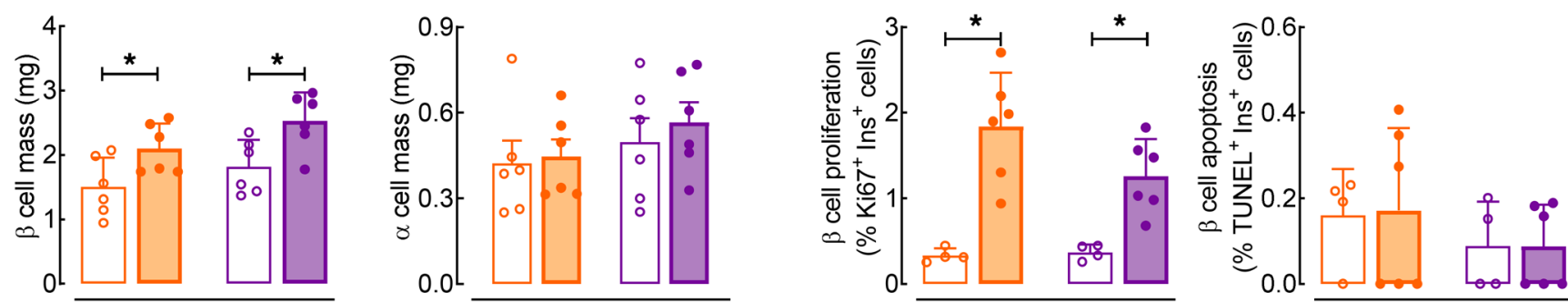

Figure 6. Selective deletion of SIc4a4 in $\beta$ cells enhances $\beta$ cell function. (A) Glucose-stimulated insulin secretion (CSIS) at $16 \mathrm{mM}$ and $4 \mathrm{mM}$ glucose concentrations in islets from $\beta-S / c 4 a 4^{+/+}\left(S / c 4 a 4^{f l / f I}+/+\right)$ and $\beta-S / c 4 a 4^{-/-}\left(S / c 4 a 4^{f / / f I} / n s 2^{C r e /+}\right)$ mice exposed to 8 weeks of chow or HFD. ${ }^{*} P<0.05$ denotes statistical significance (1-way ANOVA with Tukey's method for multiple comparisons; $n=5-9$ independent experiments from 3-5 mice). (B) Fold change in insulin secretion (16 mM vs. 4 mM glucose) (left) and insulin content (right) in $\beta-S / c 4 a 4^{+/+}\left(S / c 4 a 4^{f / / f l}+/+\right)$ and $\beta-S / c 4 a 4^{-/-}\left(S / c 4 a 4^{f / / f l} / n s 2^{\text {Cre/+}}\right.$ ) chow and HFD islets. ${ }^{*} P<0.05,{ }^{* *} P$ $<0.01,{ }^{* * *} P<0.001$ (1-way ANOVA with Tukey's method for multiple comparisons; $n=5-9$ independent experiments from 3-5 mice). (C) Slc4a4 expression in islets isolated from $\beta-S / c 4 a 4^{+/+}\left(S / c 4 a 4^{f / / f l+}+/+\right)$ mice exposed to 8 weeks of HFD after knockdown of S/c4a4. Islets were infected (96 hours) with control human

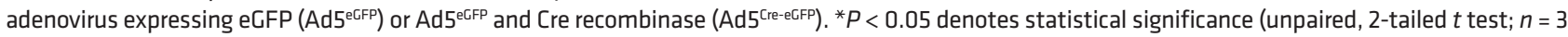
independent experiments from 3 mice). (D) Assessment of GSIS in SIc4a $4 f^{f / f f}+/+$ HFD islets infected with Ad5 $5^{\text {eCFP }}$ or Ad5 $5^{\text {Cre-eCFP; }}{ }^{*} P<0.05$ (unpaired, 2-tailed $t$ test; $n=5$ independent experiments from 3 mice). (E) Fold change in insulin secretion (16 mM vs. 4 mM glucose) (left) and insulin content (right) in Slc4a $4^{f / / f+}+/+$ HFD islets infected with Ad5 ${ }^{\text {eCFP }}$ or Ad5 $5^{\text {Cre-eCFP. }}{ }^{*} P<0.05$ (unpaired, 2-tailed $t$ test; $n=5$ independent experiments from 3 mice) (F) $\beta$ Cell mass (left) and $\alpha$ cell mass (right) in $\beta-S / c 4 a 4^{+/+}\left(S / c 4 a 4^{f / / f l}+/+\right)$ and $\beta-S / c 4 a 4^{-/-}\left(S / c 4 a 4^{f / / f I} / n s 2^{2 \text { re/ } /+}\right.$ ) mice exposed to chow or HFD. ${ }^{*} P<0.05$ (unpaired, 2-tailed $t$ test; $n=4-6$ mice per group). (G) $\beta$ Cell proliferation (left) and apoptosis (right) in $\beta-S / c 4 a 4^{+/+}\left(S / c 4 a 4^{4 / / f+}+/+\right)$ and $\beta-S / c 4 a 4^{-/-}\left(S / c 4 a 4^{f / / f} / n s 2^{\text {cre/+}}\right)$ mice exposed to chow or HFD. ${ }^{*} P<0.05$ (unpaired, 2-tailed $t$ test; $n=4-6$ independent experiments). Values represented as mean $\pm \mathrm{SEM}$.

of changes in NBCe1 in the hypothalamus (Supplemental Figure 4) and (b) utilized 2 sets of genetic controls $\left(S l c 4 a 4^{\mathrm{A} / \mathrm{l}}+/+\right.$ and ${ }^{+/} \mathrm{Ins} 2^{\mathrm{Cre} /+}$ ) for in vivo studies. Consistent with previous studies, only male mice exhibited metabolic dysfunction in response to $\operatorname{HFD}(39,40)$; thus, we carried out subsequent work in male mice

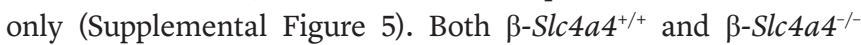
male mice exposed to the HFD exhibited comparable body mass gain and corresponding induction of insulin resistance (Figure $4 \mathrm{E}$ and Supplemental Figure 6; $P>0.05$ between genotypes). Since Slc4a4/NBCe1 plays an integral role in the regulation of both sys- temic (renal $\mathrm{HCO}_{3}{ }^{-}$absorption) and intracellular $\mathrm{pH}$, we also confirmed that blood $\mathrm{pH}$, along with blood ion and gas concentrations, were comparable between the diet and genotype groups (Supplemental Table 3; $P>0.05 \beta-S l c 4 a 4^{+/+}$vs. $\beta$-Slc4a $44^{-/-}$chow and HFD). These data illustrated that this $\beta$ cell Slc4a4-KO did not affect renal homeostatic function as observed with renal Slc4a4/NBCe1 deletion $(41,42)$. Importantly, we also confirmed reduction in islet cell $\mathrm{pH}_{\mathrm{i}}$ in $\beta$-Slc4 $44^{-/}$mice (Figure $4 \mathrm{C} ; \mathrm{P}<0.05$ vs. $\beta$-Slc $4 a 4^{+/+}$chow and HFD). Overall, we were able to successfully delete Slc4a4/NBCe1 in mouse $\beta$ cells without altering systemic $\mathrm{pH}$ and/or hypothalamic 


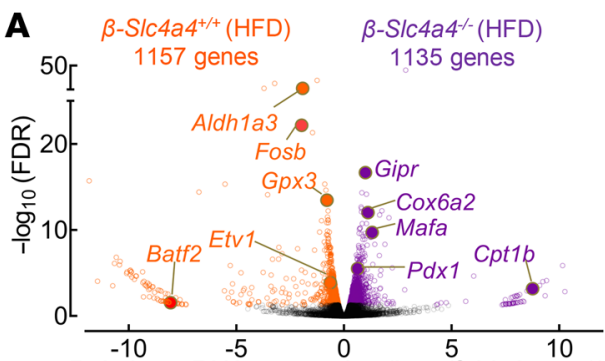

Relative mRNA expression ( $\log _{2}$ (fold change)

B

KEGG Pathways Enriched in $\beta-S / c 4 a 4^{+/+}$(HFD)

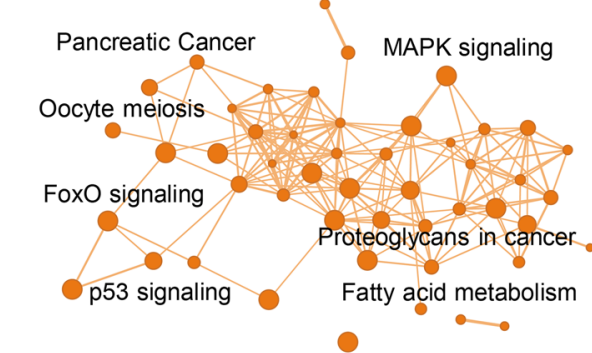

No. of genes

525

- 0

- $\bigcirc$

KEGG Pathways Enriched in $\beta$-S/c4a4 ${ }^{-/}$(HFD)

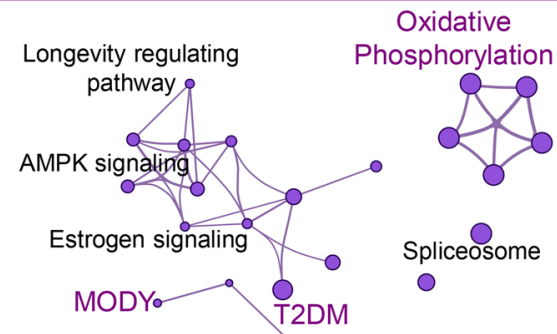

Insulin secretion

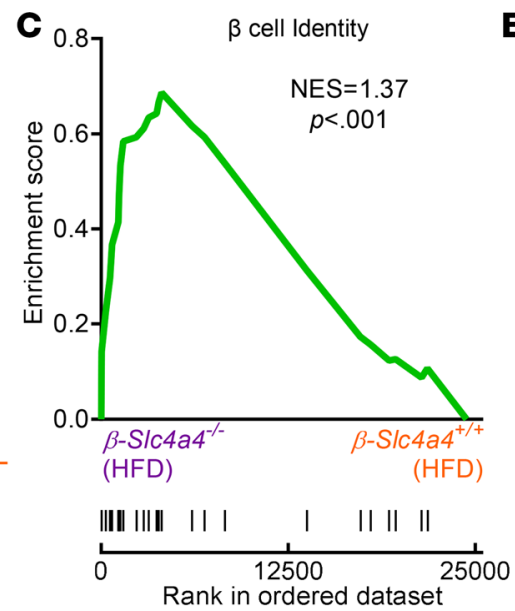

E $\beta-S / C 4 a 4^{+/+}(H F D)$

D $\beta-S / C 4 a 4^{+/+} \quad \beta-S / C 4 a 4^{-1}$

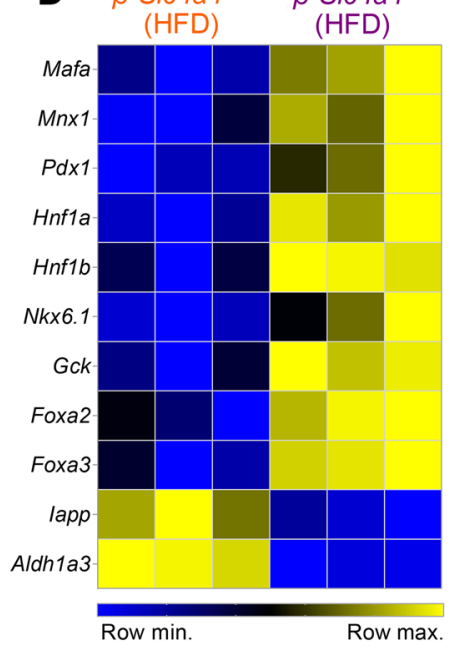

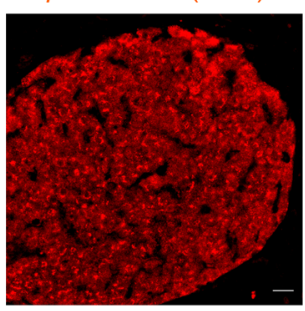
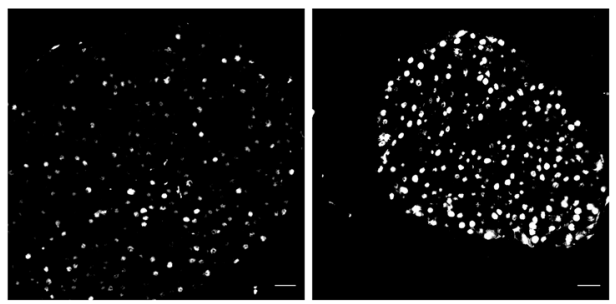

$\beta-S / c 4 a 4^{-/}($HFD)
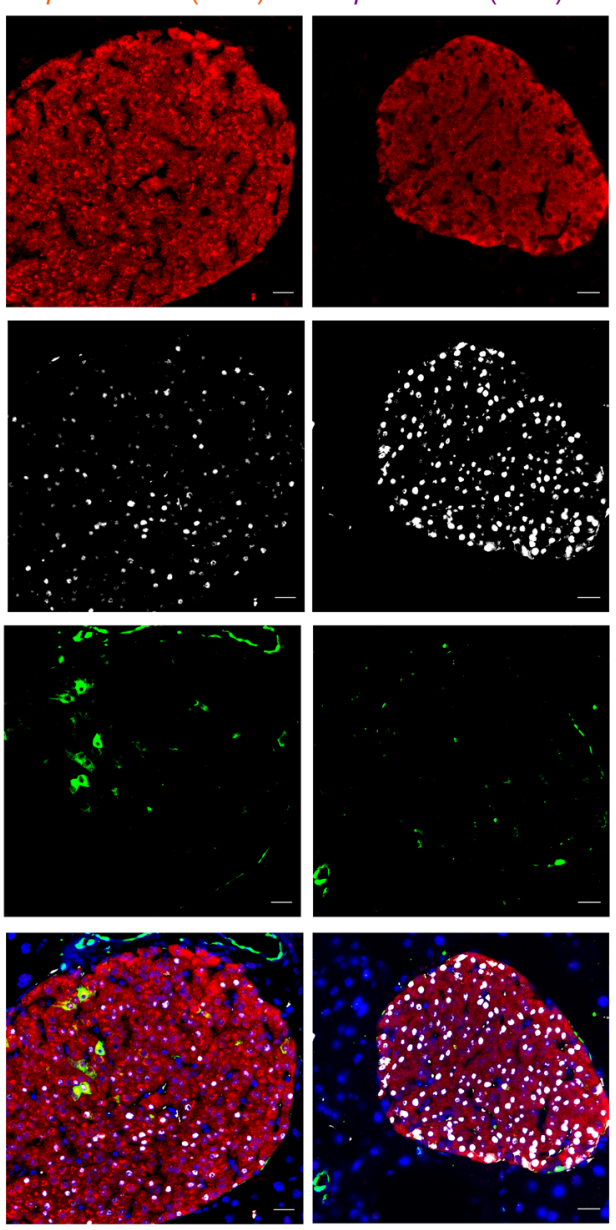

Nkx6.1 / Aldh1a3 / DAP

Figure 7. Selective deletion of SIc4a4 in $\beta$ cells preserves expression of genes regulating $\beta$ cell identity and function after exposure to a high-fat

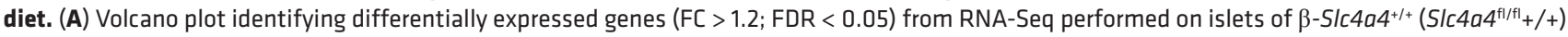
and $\beta-S / c 4 a 4^{-1-}\left(S / c 4 a 4^{f l / f I} I n s 2^{C r e /+}\right)$ mice exposed to 8 weeks of an HFD ( $n=3$ independent samples per genotype). (B) Network analysis illustrating the interaction between significantly enriched $(P<0.05)$ KEGG pathways of differentially expressed genes identified in $\beta-S / c 4 a 4^{+/+}\left(\right.$Slc $\left.4 a 4^{f / / f l}+/+\right)$ (top) and $\beta-S / c 4 a 4^{-/-}\left(S / c 4 a 4^{f / f f I} / n s 2^{C r e /+}\right)$ (bottom) HFD islets. Key pathways associated with $\beta$ cell function, identity, and stress are highlighted. (C) Gene set enrichment analysis of islet transcriptome in $\beta-S / c 4 a 4^{+/+}\left(S / c 4 a 4^{f / / f+}+/+\right)$ and $\beta-S / c 4 a 4^{-/-}\left(S / c 4 a 4^{f / / f I} / n s 2^{C r e /+}\right)$ HFD islets for $\beta$ cell identity (KEGG - maturity onset of diabetes in the young pathway; $P<0.001$ ) (D) Heatmap visualization of transcriptional changes of genes encoding $\beta$ cell identity and dedifferentiation

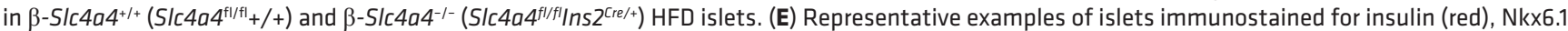

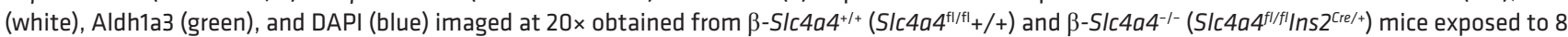
weeks of an HFD. Scale bars: $20 \mu \mathrm{m}$ in all images. Images are representative of $n=3$ mice per group.

NBCe1 expression, allowing us to probe the role of this transporter in the regulation of $\beta$ cell functional and transcriptional identity under diabetogenic conditions in vivo.

Selective deletion of Slc4a4 in $\beta$ cells confers protection from $H F D$-induced glucose intolerance and $\beta$ cell dysfunction. We next addressed whether $\beta$ cell-selective deletion of Slc $4 a 4 / \mathrm{NBCe} 1$ confers protection against HFD-induced glucose intolerance and $\beta$ cell dysfunction (Figure 5). Prior to administration of the HFD (e.g., week 0), $\beta$-Slc4a $4^{+/+}$versus $\beta$-Slc4a $44^{-/-}$mice displayed comparable glucose tolerance and insulin secretory response, thus confirming absence of potential "off-target" metabolic effects of Slc4a4 deletion using the RIP-Cre model (Figure 5A; $P>0.05 \beta-S l c 4 a 4^{+/+}$ vs. $\left.\beta-S l c 4 a 4^{--}\right)$. After 4 and 8 weeks of the HFD, $\beta-S l c 4 a 4^{+/+}$mice exhibited glucose intolerance evident by an approximately $50 \%$ increase in glucose AUC during i.p. glucose tolerance testing (ipGTT) (Figure 5, B and C; $P<0.05$ vs. $\beta-S l c 4 a 4^{+/+}$chow). However, HFD-fed $\beta$-Slc4a4 $4^{-/-}$mice demonstrated the same degree of glucose tolerance as their chow-fed counterparts, indicating protection from HFD-induced glucose intolerance (Figure 5, B and $C ; P>0.05$ vs. $\beta$-Slc4a4 $/-$ chow-fed and $P<0.05$ vs. HFD-fed $\beta$-Slc $\left.4 a 4^{+/+}\right)$. Enhanced glucose tolerance in HFD-fed $\beta$-Slc $4 a 4^{-/-}$ mice was attributed to preservation of the in vivo insulin response at weeks 4 and 8 (Figure 5, B and C) rather than alterations in insulin sensitivity (Supplemental Figure 6). 
A Oxidative Phosphorylation
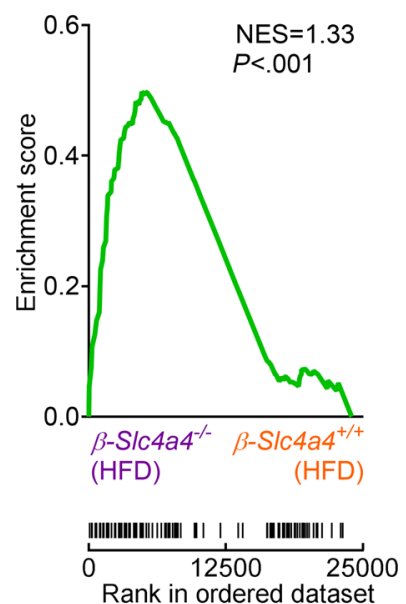

C

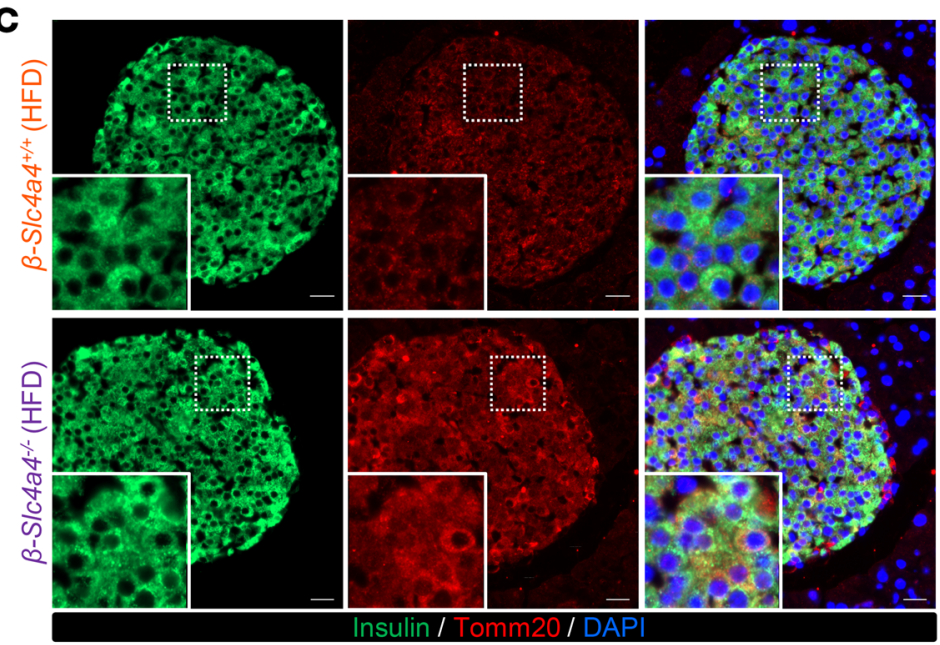

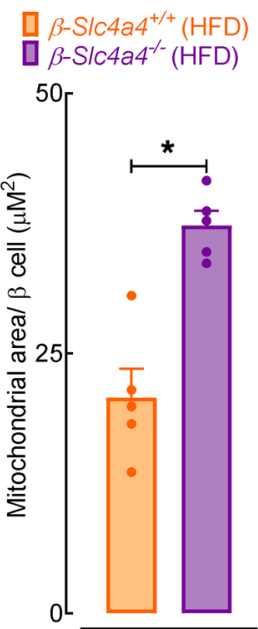

B

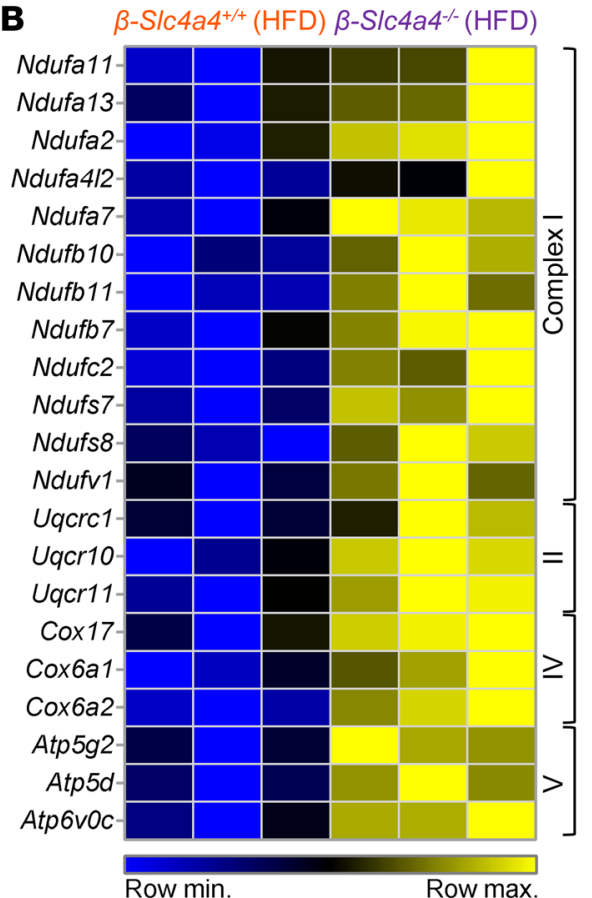

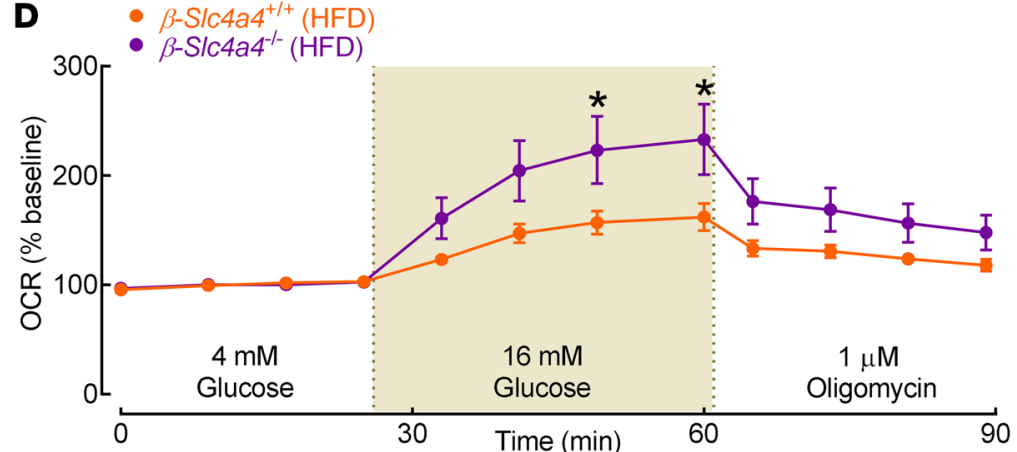

E $\quad\left[\beta-S / C 4 a 4^{+/+}(H F D)\right.$

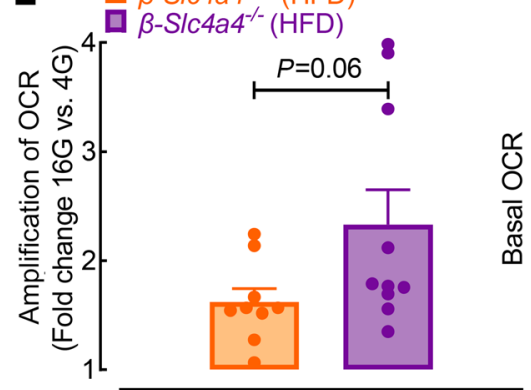

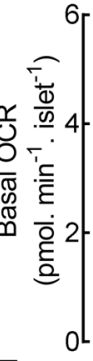

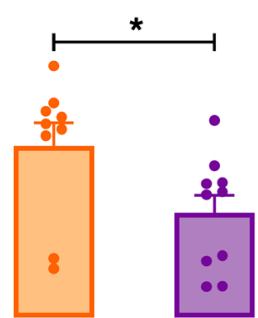

Figure 8. Selective deletion of SIc4a4 in $\beta$ cells preserves mitochondrial function and area after exposure to a high-fat diet. (A) Gene set enrichment analysis of islet transcriptome in $\beta-S I c 4 a 4^{+/+}\left(S / c 4 a 4^{f / / f l}+/+\right)$ and $\beta-S / c 4 a 4^{-/-}\left(S / c 4 a 4^{f / f f l} / n s 2^{C r e /+}\right)$ HFD islets for KEGC oxidative phosphorylation pathway (P $<0.001$ ). (B) Heatmap visualization of transcriptional changes of genes encoding mitochondrial complexes in $\beta$-S/c $4 a 4^{+/+}$and $\beta$-S/c4a4 ${ }^{-/-}$HFD islets. (C) Representative examples of mouse pancreatic sections immunostained for the outer mitochondrial membrane receptor Tomm20 (red), insulin (green), and nuclear marker DAPI (blue) obtained from $\beta-S / c 4 a 4^{+/+}\left(S / c 4 a 4^{f / / f 1}+/+\right)$ and $\beta-S / c 4 a 4^{-/-}\left(S / c 4 a 4^{f / / f I} / n s 2^{c r e /+}\right)$ mice exposed to 8 weeks of HFD and imaged at $20 \times$ magnification. Scale bars: $20 \mu \mathrm{m}$ in all images (left). Quantification of mitochondrial area per $\beta$ cell as represented by the fraction of Tomm20 pixels relative to the number of insulin $\beta$ cells (right). ${ }^{*} P<0.05$ denotes statistical significance (unpaired, 2 -tailed $t$ test; $n=5$ mice per group). (D) $0 x y g e n$ consumption rate (OCR) normalized to basal OCR measured by Seahorse XFe24 in isolated islets from $\beta-S / c 4 a 4^{+/+}\left(S / c 4 a 4^{f l / f 1}+/+\right)$ and $\beta-S / c 4 a 4^{-/-}\left(S / c 4 a 4^{f l / f l} / n s 2^{C r e /+}\right.$ ) mice exposed to 8 weeks of HFD. ${ }^{*} P<0.05$ denotes statistical significance (unpaired $t$ test with Holm-Sidak method for multiple comparisons; $n=9-10$ independent experiments from $\geq 4$ mice/group). (E) Glucose-stimulated OCR was calculated as the ratio of $16 \mathrm{mM}$ glucose AUC to basal $4 \mathrm{mM}$ glucose AUC (left). Basal OCR quantified as mean OCR in $4 \mathrm{mM}$ glucose normalized to islet number (right). ${ }^{*} P<0.05$ denotes statistical significance (unpaired 2 -way $t$ test; $n=9-10$ independent experiments from $\geq 4$ mice/group). All values reported are represented as mean $\pm \mathrm{SEM}$.

To examine in vitro $\beta$ cell function, we utilized GSIS during islet static incubations and islet perifusion assays from chow- and HFD-fed $\beta$-Slc4a $4^{-/-}$and control $\beta-S l c 4 a 4^{+/+}$mice (Figure 6, A-E and Supplemental Figure 7). Islets isolated from HFD-fed control $\beta$-Slc $4 a 4^{+/+}$mice exhibited characteristic features of $\beta$ cell dysfunc- tion: increased basal nonstimulatory insulin release, attenuated insulin response to glucose, and consequently, impaired glucose stimulation index (Figure 6, A and B; $P<0.05$ for basal insulin release and stimulation index for $\beta$-Slc $4 a 4^{+/+}$chow vs. $\beta$-Slc $4 a 4^{+/+}$ HFD mice). Importantly, $\beta$ cell deletion of Slc4a4 attenuated 
HFD-mediated insulin secretory dysfunction, highlighted by a complete reversal of basal insulin hypersecretion and corresponding increase in the glucose stimulation index in HFD-fed $\beta$-Slc $4 a 4^{-/-}$versus control $\beta$-Slc $4 a 4^{+/+}$mice (Figure 6, A and B and Supplemental Figure 7; $P<0.05$ for basal insulin release and stimulation index for $\beta-S l c 4 a 4^{+/+}$vs. $\beta$-Slc $4 a 4^{-/-}$HFD mice). To further confirm the beneficial effects of $S l c 4 a 4$ deletion on $\beta$ cell function, we used an adenovirus for Cre recombinase (Ad5 $5^{\text {Cre-eGFP) }}$ to achieve the recombination of $\operatorname{Slc} 4 a 4$ floxed alleles in islets isolated from HFD-fed Slc $4 a 4^{f l / f l}$ mice independent of RIP-Cre-driven recombination (Figure 6, C-E). Consistently, addition of an adenovirus for Cre recombinase (versus $\mathrm{Ad} 5^{\mathrm{eGFP}}$ control virus) resulted in a robust suppression of Slc4a4 mRNA and corresponding enhancement of GSIS response in islets isolated from HFD-fed Slc4a $44^{f / f l}$ mice (Figure 6, C-E; $P<0.05$ for glucose stimulation index for Ad5 ${ }^{\text {Cre-egFP }}$ vs. control Ad5 $\left.5^{\text {eGFP }}\right)$. Finally, enhanced $\beta$ cell function and glucose tolerance in HFD-fed $\beta$-Slc $4 a 4^{-/-}$mice were not attributed to alterations in the endocrine cell numbers signified by comparable $\beta$ and/or $\alpha$ cell mass, proliferation, apoptosis, and in vitro islet response to $\mathrm{KCl}$ stimulation in $\beta-S l c 4 a 4^{-/-}$versus control $\beta$-Slc $4 a 4^{+/+}$mice (Figure 6, F and G and Supplemental Figure 8).

Selective deletion of Slc4a4 in $\beta$ cells preserves expression of genes regulating $\beta$ cell identity and function after exposure to the HFD. To examine how changes in the transcriptional landscape relate to enhanced $\beta$ cell function in $\beta$-Slc4a4 ${ }^{-/}$HFD mice, we performed RNA isolation followed by RNA-Seq from $\beta-S l c 4 a 4^{+/+}$and $\beta$-Slc4a4 ${ }^{-/-}$HFD islets (Figure 7 and Supplemental Figure 9; $n=$ 3 biological repeats per condition, GEO GSE179284). RNA-Seq revealed approximately 1000 differentially expressed transcripts annotated to divergent (KEGG) biological pathways in $\beta$-Slc4a $44^{+/+}$ and $\beta$-Slc $4 a 4^{-/-}$HFD islets (Figure 7, A and B and Supplemental Table 4). Specifically, $\beta$-Slc $4 a 4^{+/+}$HFD islets differentially expressed transcripts enriched for pathways regulating $\beta$ cell dysfunction and stress (e.g., MAPK signaling, p53 signaling, FOXO signaling), while $\beta$-Slc4 $44^{-/-}$HFD islets were enriched for pathways important for the regulation of $\beta$ cell function and identity and mitochondrial function (e.g., insulin secretion, MODY, oxidative phosphorylation; Figure 7B). Further probing of these transcriptomic changes by gene set enrichment analysis (GSEA) revealed that 1 of the top 2 enriched pathways in $\beta-S l c 4 a 4^{-/-}$HFD islets regulated $\beta$ cell identity with a normalized enrichment score of 1.37 (Figure $7 C ; P<0.001)$. These findings, confirmed by quantitative real-time PCR (qRT-PCR), demonstrated significant enhancement in mRNA expression of key $\beta$ cell identity transcription factors, Nkx6.1, Mafa, and $P d x 1$, in $\beta$-Slc4a4 $4^{-/-}$HFD mice (Figure 7D and Supplemental Figure 10; $P<0.05$ vs. $\beta$-Slc $4 a 4^{+/+}$HFD). Moreover, genes associated with $\beta$ cell stress and loss of transcriptional identity (e.g., Iapp, Aldh1a3) were markedly suppressed in $\beta$-Slc4a $44^{-/-}$HFD mice (Figure 7D and Supplemental Figure $10 ; P<0.05$ vs. $\beta-S l c 4 a 4^{+/+}$HFD). Utilizing immunofluorescence analysis, we confirmed reduction of Nkx6.1 and increase in Aldh1a3 immunoreactivity in $\beta-S l c 4 a 4^{+/+}$ HFD $\beta$ cells compared with $\beta$-Slc $4 a 4^{-/-}$HFD (Figure 7E).

Selective deletion of Slc4a4 in $\beta$ cells preserves mitochondrial function and area after exposure to HFD. Previous studies have suggested that loss of $\beta$ cell identity and function is strongly associated with diminished mitochondrial function and consequent divergence of glycolytic flux from oxidation phosphorylation toward lactic acid production $(6,8,43)$. Since decreased $\mathrm{pH}_{\mathrm{i}}$ promotes mitochondrial oxidative phosphorylation partly through the regulation of NBCe1 activity (44), we hypothesized that the enhancement in $\beta$ cell function in HFD-fed $\beta$-Slc $4 a 4^{-/-}$islets was due, in part, to maintenance of mitochondrial function and oxidative phosphorylation. GSEA revealed oxidative phosphorylation as a significantly enriched pathway in $\beta$-Slc $4 a 4^{-/-}$HFD transcripts with a normalized enrichment score of 1.33 (Figure 8A; $P$ $<0.001)$. Indeed, transcripts regulating mitochondrial complexes I, III, IV, and V were enriched in $\beta-S l c 4 a 4^{-/-}$HFD islets (Figure 8B; FDR $<0.05$ vs. $\beta-S l c 4 a 4^{+/+}$HFD), which was confirmed by qRTPCR (Supplemental Figure 10; $P<0.05$ vs. $\beta-S l c 4 a 4^{+/+}$HFD). Interestingly, key transcripts regulating mitochondrial function were also enriched in isolated T2DM human islets treated in vitro with NBCe1 inhibitor S0859 (Supplemental Figure 10; $P<0.05$ vs. vehicle). Consistent with changes to $\beta$ cell function and islet transcriptome, we also observed an increase in $\beta$ cell mitochondrial area/network in $\beta$-Slc $4 a 4^{-/-}$versus $\beta$-Slc $4 a 4^{+/+}$HFD-fed mice, which was assessed with Tomm20 immunostaining (Figure 8C; $P$ $<0.05$ vs. $\beta-S l c 4 a 4^{+/+}$HFD). Moreover, $\beta$-Slc $4 a 4^{-/-}$HFD islets were also characterized by enhanced glucose-stimulated oxygen consumption rate (OCR) and a significant reduction $(\sim 60 \%)$ in basal OCR (Figure 8, D and E; $P<0.05$ vs. $\beta-S l c 4 a 4^{+/+}$HFD).

\section{Discussion}

The etiology of $\beta$ cell dysfunction in T2DM can be distilled to the loss of GSIS in response to the exposure of metabolic, oxidative, and inflammatory stressors $(45,46)$. Although a number of key signaling cascades have been identified to mediate these effects, the root consequence of these stressors can be traced to reprogramming of the tightly controlled $\beta$ cell transcriptional landscape through dysregulation of key transcription factors associated with the $\beta$ cell's core identity $(6,33,34,43,47)$. Indeed, studies have demonstrated that central to mature $\beta$ cell function is the activation of key machinery associated with glucose sensing, oxidative metabolism, and insulin biogenesis/secretion with simultaneous repression of ubiquitously expressed genes that would otherwise impede these processes $(48,49)$. However, the functional decline, transcriptional reprogramming, and dedifferentiation associated with the T2DM $\beta$ cell is not without purpose. For instance, the well-characterized transcriptional and metabolic shift from oxidative to glycolytic metabolism in T2DM $\beta$ cells provides a flexible survival advantage in response to diabetogenic stressors such as hypoxia, ER stress, and aberrant $\mathrm{Ca}^{2+}$ signaling (50-52). Although great strides have been made in characterizing key transcriptional and functional changes in the T2DM $\beta$ cell, further studies are needed to advance our understanding of these processes.

Herein, the current investigation aimed to delineate the role of a previously uncharacterized transcript enriched in T2DM $\beta$ cells, $S L C 4 A 4$, and its protein product, the electrogenic $\mathrm{Na}^{+}-\mathrm{HCO}_{3}{ }^{-}$cotransporter NBCe1. Although there are limited reports characterizing changes in $\mathrm{HCO}_{3}^{-}$transport in T2DM $\beta$ cells, there is accumulating evidence that $S L C 4 A 4 / \mathrm{NBCe} 1$ plays a key role in the development and progression of cancer $(22,52)$. These data become relevant as studies suggest that the transcriptional and functional alterations in T2DM $\beta$ cells resemble changes observed in cancer cells (5-7), thus providing $\beta$ cells a survival advantage at the expense of reduced 
insulin secretory function. For example, 2 recently identified cancer pluripotency genes (e.g., PFKFB3 and ALDH1A3) are characteristically induced in T2DM $\beta$ cells where they purportedly contribute to impaired GSIS $(5,8)$. Consistently, the upregulation/activation of key $\mathrm{HCO}_{3}^{-}$transporters (e.g., NBCe1) and acid extruders (e.g., NHE1, MCT1) in cancer promotes increased glycolytic flux, lactate generation, and in turn proliferative capacity through (a) increased cellular buffering (known as "buffering power") to neutralize an increase in metabolic acid formation and (b) increased activity of key glycolytic enzymes (e.g., LDHA, PFK) due to a more permissive alkaline environment $(10,53,54)$. Although counterintuitive, activation of these key transporters has been shown to create a "reverse $\mathrm{pH}$ gradient" whereby $\mathrm{H}^{+}$created in glycolytic metabolism and lactate production acidify the extracellular space, with $\mathrm{pH}_{\mathrm{i}}$ becoming increasingly alkaline through continued $\mathrm{H}^{+}$export and $\mathrm{HCO}_{3}^{-}$import $(9,21,55)$. Indeed, T2DM $\beta$ cells exhibit these transcriptional and phenotypical metabolic changes, yet there has been little study into whether there are concomitant changes in $\mathrm{pH}_{\mathrm{i}}$ regulation and potential functional consequences of such changes in T2DM.

In our study, we found that $S L C 4 A 4 / \mathrm{NBCe} 1$ induction in T2DM $\beta$ cells was associated with basal intracellular alkalinization and $\beta$ cell dysfunction. Both were reversed by pharmacological inhibition of NBCe1 in T2DM and genetic deletion of Slc4a4 in response to diet-induced obesity. This is consistent with a series of eloquent studies from the Piston group demonstrating that diabetic mouse islets had alterations in $\mathrm{pH}$ regulation, notably basal intracellular alkalinization in diabetic KK islets (26). Moreover, intracellular acidification via pharmacological inhibition of $\mathrm{Na}^{+} / \mathrm{H}^{+}$exchange was able to restore GSIS in diabetic mice in vivo and in vitro through enhanced time-dependent potentiation $(24,26,27)$. In addition, the recent observation that the alkaline $\mathrm{pH}-$ activated 2-pore domain of the TALK- $1 \mathrm{~K}^{+}$channel limits insulin secretion, and its activation is associated with $\mathrm{T} 2 \mathrm{DM}$ risk, further supports these findings (56). The $\beta$ cell-selective NBCe1 deletion ( $\beta$-Slc4a4 $4^{-/}$) appears to protect against $\beta$ cell dysfunction in diet-induced obesity and likely T2DM. These protections are through enhancement of mitochondrial subunit gene expression and oxidative glucose metabolism and were associated with suppression of markers of dedifferentiation and increased glycolytic flux, such as Aldh1a3 and Adh7. This model is consistent with findings that failing, immature, dedifferentiating, and T2DM $\beta$ cells exhibit downregulation in the expression of many genes and proteins (e.g., NDUFA, COX, ATP6V) key to mitochondrial function $(3,57,58)$. Interestingly, SLC4A4 transcript levels are also induced in immature $\beta$ cells ( $~ 4.2$-fold vs. mature $\beta$ cells), Aldh $^{+} \beta$ cells ( $\sim 4.0$-fold vs. Aldh $\beta$ cells), and stem cell-derived $\beta$ cells $(\sim 2.7$-fold vs. human $\beta$ cells), all models of reduced mitochondrial function $(8,59,60)$.

It is important to note that the elucidation of mechanisms underlying $\beta$ cell dedifferentiation in $\mathrm{T} 2 \mathrm{DM}$ remains an area of active investigation. Indeed, several molecular mechanisms mediating dedifferentiation of $\beta$ cells have been proposed, which include (but are not limited to) cellular hypoxia (5), mechanical stress (61), and DNA methylation (62). Our current data suggest that the induction of $S l c 4 a 4 / \mathrm{NBCe} 1$ plays a contributory role in the $\beta$ cell dedifferentiation process, likely through $\beta$ cell alkalinization. Although additional studies are warranted, $\beta$ cell alkalinization may permit metabolic switching from mainly oxidative metabolism to increased glycolytic flux and lactate production by increasing $\beta$ cell buffering capacity in response to metabolic stress
$(10,53,54)$. In addition, changes in $\mathrm{pH}_{\mathrm{i}}$ may also contribute to epigenetic reprogramming of global gene expression as well as regulation of DNA and protein synthesis $(9,11,12)$. Finally, it is also plausible that increased expression of SLC4A4/NBCe1 may affect $\beta$ cell transcriptome/function independent of $\mathrm{pH}_{\mathrm{i}}$ alterations. For example, members of the SLC4 family of transporters (e.g., $S L C 4 A 1 /$ Band 3) play a role in the regulation of cellular shape, membrane stability, and cytoskeleton dynamics, all of which are critical determinants of the cell differentiation process (63).

Overall, we currently have limited insight into how $\mathrm{HCO}_{3}^{-}$ transport and in turn $\mathrm{pH}_{\mathrm{i}}$ regulation are altered in T2DM $\beta$ cells. While classical studies pioneered by Henquin and colleagues have elucidated that extracellular glucose induces acute/transient (e.g., 0-10 minutes) intracellular alkalinization, likely through $\mathrm{HCO}_{3}^{-}$-mediated transport mechanisms, previous work has mainly focused on the acute role of insulin secretagogues on islet $\mathrm{pH}_{\mathrm{i}}(64$, 65). Our current work builds on early studies that demonstrated $\beta$ cell alkalinization in islets of multiple mouse models of T2DM and the corresponding enhancement of insulin secretion after administration of $\mathrm{pH}_{\mathrm{i}}$-lowering agents $(26,27)$. Interestingly, it was recently noted that chronic hyperglycemia in vitro induces $\beta$ cell alkalinization (66). Collectively, our current studies support the notion that the diabetogenic state is associated with $\beta$ cell cytosolic alkalinization, which we have now shown is attributed in part to the induction of $S L C 4 A 4 / \mathrm{NBCe} 1$ expression/activity in T2DM. Moreover, our studies also demonstrated that the induction of SLC4A4/NBCe1 in $\beta$ cells is driven by exposure to specific diabetogenic stressors such as ER stress/lipotoxicity. Importantly, these observations are consistent with the analysis of the SLC4A4 promoter landscape and previous studies that examined mechanisms regulating transcriptional control of $S L C 4 A 4 / \mathrm{NBCe} 1$ expression $(12,13)$.

Pharmacological inhibition of NBCe1 with S0859 was able to lower $\mathrm{pH}_{\mathrm{i}}$ and enhance GSIS in T2DM human islets. Thus, inhibition of NBCe1 may be considered as a potential strategy to counteract $\beta$ cell failure in T2DM. Indeed, NBCe1 inhibition has been shown to be an effective cardioprotective agent in animal and cell models of ischemia/reperfusion injury (67) and prevents malignant dedifferentiation of glial cells (68). Although the potency, NBCe1 selectivity, and $\mathrm{pH}_{\mathrm{i}}$-lowering capacity of $\mathrm{S} 0859$ has been extensively studied and validated (69), the pharmacological action of S0859 in human $\beta$ cells/islets has not been formally studied. Additionally, the systemic role of NBCe1 in global acid-base homeostasis impedes systemic delivery of NBCe 1 inhibitors as a potential treatment option for $\beta$ cell failure in T2DM (19). In this regard, efforts in $\beta$ cell-targeted drug delivery using glucagon-like peptide- 1 fusion peptides, for instance, could allow for selective targeting of $\mathrm{pH}$-regulating pathways as a novel therapeutic target in T2DM (70). In summary, here we provided evidence of changes to $\mathrm{Na}^{+} \mathrm{HCO}_{3}^{-}$cotransport via $\mathrm{NBCe} 1$ in T2DM $\beta$ cells and demonstrated that inhibition of this pathway may provide a promising therapeutic approach to address $\beta$ cell dysfunction in response to obesity and T2DM.

\section{Methods}

Human pancreas and islet studies. Human pancreas was procured from the Mayo Clinic autopsy archives with approval from the Institutional Research Biosafety Board. Isolated human islets were obtained from Prodo Laboratories or the Integrated Islet Distribu- 
tion Program. Pancreatic sections and/or isolated islets were immunostained for insulin (Abcam, ab7842), NBCe1 (Santa Cruz Biotechnology, sc-515543; antiserum K1A; ref. 71), glucagon (Abcam, ab92517; MilliporeSigma, G2654), NKX6.1 (Developmental Studies Hybridoma Bank, F55A12), and NHE1 (Santa Cruz Biotechnology, sc-136239), with Vectashield-DAPI mounting medium (Vector Laboratories). Blinded slides were viewed, imaged, and analyzed using an Axios Observer Z1 microscope (Carl Zeiss Microscopy) and ZenPro software (Carl Zeiss Microscopy). NBCe1 expression was quantified using Image (NIH) as the fraction of either NBCe1-insulin or NBCe1-glucagon double-positive pixels per the total number of insulin- or glucagon-positive pixels following background subtraction. To quantify the percentage of insulin-positive $\beta$ cells with nuclear and/ or cytoplasmic NKX6.1 localization, at least $250 \beta$ cells per pancreatic section were examined in detail and counted at $20 \times$ magnification for the presence of NKX6.1 immunoreactivity specifically in the nucleus (nuclear) or with the presence of cytoplasmic NKX6.1 (cytoplasmic). These cells were then subclassified depending on the presence or absence of NBCe1 following background subtraction. For NBCe1 inhibitor studies, groups of 150 to 200 manually picked human islets were treated with either vehicle (DMSO) or 72 hours of chronic $30 \mu \mathrm{M}$ S0859 treatment (Biovision, 9425). Groups of 10 to 15 islets were then subjected to a GSIS assay, which was assessed by static incubation in Krebs Ringer bicarbonate buffer (KRBH; pH 7.4) at basal (4 mM glucose per 30 minutes) and hyperglycemic conditions (16 mM glucose per 30 minutes) containing either vehicle (DMSO) or $30 \mu \mathrm{M}$ S0859.

Animal models. Floxed Slc4a4 mice were obtained from the University of Cincinnati (35) and crossed with mice expressing Cre driven by RIP (The Jackson Laboratory, Tg [Ins2-cre] 25Mgn/J). To identify resulting offspring, mice were first genotyped for the presence of the floxed or wild-type Slc4a4 allele using a PCR-based method. Primers designed for the flanking the proximal LoxP site (F; $5^{\prime}$-TGGTGGCTTAAATTGCAAATGGC-3'; R: 5'-CATAACCCACTAAGTCCAGTACG-3') yield a $223 \mathrm{bp}$ product for the floxed allele and $176 \mathrm{bp}$ for the wild-type allele (35). The presence or absence of Ins $2^{\mathrm{Cre} / \mathrm{+}}$ was identified by primers designed for Cre-recombinase (F 5'-GCGGTCTGGCAGTAAAAACTATC-3'; R 5'-GTGAAACAGCATTGCTGTCACTT-3'). All animals were housed under standard 12-hour light/12-hour dark cycle and provided with either ad libitum chow diet (14\% fat, 32\% protein, and $54 \%$ carbohydrates; Harlan Laboratories) or HFD (60\% fat, $20 \%$ protein, 20\% carbohydrates; Research Diets).

Metabolic in vivo mouse studies. All metabolic studies were conducted in mice after brief fasting (2 hours). Glucose tolerance testing was performed by i.p. injection of $1 \mathrm{~g} / \mathrm{kg}$ body weight of $50 \%$ dextrose. Blood glucose was measured at 0, 15, 30, 45, 60, and 90 minutes after dextrose injection with simultaneous measurement of plasma insulin at baseline and 15 minutes after glucose administration. Insulin tolerance was assessed after i.p. injection of $0.75 \mathrm{mU} / \mathrm{g}$ body weight of insulin (Novolin, Novo Nordisk) with subsequent measurement of blood glucose at baseline and then every 10 minutes for 1 hour after injection (72).

Mouse islet isolation and measurements of GSIS. Pancreatic mouse islets were isolated using the collagenase method as previously described (72) and allowed to recover overnight incubated in standard RPMI 1640 medium supplemented with $10 \% \mathrm{FBS}$ at $37^{\circ} \mathrm{C}$. GSIS was performed as described above.
Adenoviral infection of mouse islets. Isolated mouse islets were washed with $1 \times$ PBS and infected with $2 \times 10^{7} \mathrm{PFU}$ per 100 islets of either (a) Ad5 expressing eGFP reporter and Cre recombinase (Ad5 $5^{\text {Cre-eGFP }}$; Vector Biolabs) to delete Slc4a4 in vitro or (b) control human adenovirus type 5 expressing eGFP reporter (Ad5 ${ }^{\text {eGFP }}$ ). After 24 hours, islets were washed in standard RPMI 1640 media and incubated for an additional 72 hours at $37^{\circ} \mathrm{C}$ and $5 \% \mathrm{CO}_{2}$.

Intracellular $\mathrm{pH}$ measurement. Isolated islets were recovered overnight in standard conditions and subsequently transferred to basal KRBH. A pH-sensitive dye, SNARF5 (Invitrogen, Thermo Fisher Scientific), was added at a concentration of $5 \mu \mathrm{M}$ for 1 hour at $37^{\circ} \mathrm{C}$ and $5 \% \mathrm{CO}_{2}$. Islets were immediately monitored using an LSM510 confocal laser-scanning microscope (Zeiss) on a warmed and humidified stage $\left(37^{\circ} \mathrm{C} ; 5 \% \mathrm{CO}_{2}\right)$. Images were obtained by collecting emission fluorescence in the bandwidth ranges of 580 to $620 \mathrm{~nm}$ and 630 to $670 \mathrm{~nm}$, from an excitation with an argon laser at $514 \mathrm{~nm}$. Image J (NIH) was used to calculate the fluorescence ratio from well-loaded cellular regions of interest in the islet. Absolute $\mathrm{pH}_{\mathrm{i}}$ was obtained by calibrating known $\mathrm{pH}_{\mathrm{i}}(\mathrm{pH} 6$ to 8$)$ to a given islet cell fluorescence ratio using a $20 \mu \mathrm{M}$ nigericin and $100 \mathrm{mM} \mathrm{K}^{+}$solution (32).

Mouse histological studies. Mice were euthanized, and the pancreas and brain were subsequently extracted, fixed in $4 \%$ paraformaldehyde, and embedded in paraffin. Sections were then immunostained for insulin (Abcam, ab7842 or R\&D Systems, MAB1417), glucagon (Abcam, ab92517), Aldh1a3 (Novus Biologicals, NBP2-15339), Nkx6.1 (Developmental Studies Hybridoma Bank, F55A12), mitochondrial membrane marker Tomm20 (GeneTex, GTX32928), replication marker Ki67 (BD Pharmingen, 550609), and apoptosis marker TUNEL (Roche, 12156792910). Blinded slides were viewed, imaged, and analyzed using an Axios Observer Z1 microscope (Carl Zeiss Microscopy) and ZenPro software (Carl Zeiss Microscopy). $\beta$ Cell and $\alpha$ cell mass were calculated as the fraction of insulin/glucagon-positive pancreatic area multiplied by the pancreatic mass. $\beta$ Cell replication and apoptosis were quantified as the fraction of Ki67/TUNEL-positive $\beta$ cells per total $\beta$ cell number. $\beta$ Cell mitochondrial area was quantified as the number of Tomm20-positive pixels per total $\beta$ cell number.

Cell culture and in vitro treatment with diabetogenic stressors. Mouse INsulinoma 6 cells (AddexBio, MIN6) were cultured in DMEM containing $4.5 \mathrm{~g} / \mathrm{L}$ glucose and supplemented with $15 \% \mathrm{FBS}, 1 \mathrm{mM}$ sodium pyruvate, and $50 \mu \mathrm{M} \beta$-mercaptoethanol. For treatment of MIN6 cells, 1 $\times 10^{6}$ cells were seeded and allowed to recover overnight. Subsequently, the media was replaced with either media containing vehicle (DMSO) or the following: $200 \mathrm{nM}$ doxorubicin (Tocris Bioscience, 225210), 1 $\mu \mathrm{M}$ tunicamycin (MP Biomedicals, 150028), $1 \mu \mathrm{M}$ thapsigargin (MilliporeSigma, T9033), $250 \mu \mathrm{M} \mathrm{H}_{2} \mathrm{O}_{2}$ (MilliporeSigma, H1009), Cytomix containing $0.2 \mathrm{ng} / \mathrm{mL}$ IL-1 $\beta$ (R\&D Systems, 201-LB-005/CF), $10 \mathrm{ng} /$ mL TNF- $\alpha$ (R\&D Systems, 210-TA-020/CF), 10 ng/mL IFN- $\gamma$ (R\&D Systems, $585-\mathrm{IF}-100 / \mathrm{CF}$ ), 4 ng/mL TGF- $\beta$ (R\&D Systems, 240-B-002/ $\mathrm{CF}$ ), or $0.5 \mathrm{mM}$ palmitate (MilliporeSigma, P9767) for 2, 4, 8, or 24 hours prior to RNA isolation. For treatment of isolated mouse and human islets, islets were recovered overnight in standard media. Groups of 100 to 150 mouse and human islets were handpicked and then treated with either media containing vehicle (DMSO), $1 \mu \mathrm{M}$ thapsigargin, or $0.5 \mathrm{mM}$ palmitate for 8 or 24 hours prior to RNA isolation or $\mathrm{pH}$ imaging.

Mitochondrial function measurement. Isolated islets were recovered overnight in standard conditions, and 50 islets per well were seeded onto an Islet Capture XF24 plate (Agilent Technologies). Prior to the assay, 
islets were equilibrated at $37^{\circ} \mathrm{C}$ in basal media ( $4 \mathrm{mM}$ glucose) for 1 hour. Mitochondrial function was determined according to the manufacturer's instructions for pancreatic islets using the Seahorse XFe24 Analyzer (Agilent Technologies). OCR, measured every 8 to 9 minutes by fluorescent probes, was determined in the following sequence: baseline, $16 \mathrm{mM}$ glucose, and $5 \mu \mathrm{M}$ oligomycin over 4 cycles each. Measurements were normalized to baseline OCR. Glucose-stimulated OCR was calculated as the AUC following application of $16 \mathrm{mM}$ glucose divided by the basal AUC.

qRT-PCR analysis. Total RNA was isolated using the RNeasy Mini kit (QIAGEN). cDNA was transcribed from 200 to 300 ng of RNA with the iScript cDNA Synthesis kit (Bio-Rad), and the resulting cDNA was mixed with SYBR Green Master Mix (ABI) with gene-specific primers (Supplemental Table 4) for a total reaction volume of $25 \mu \mathrm{L}$. qRT-PCR analysis was performed using the ABI StepOnePlus Real-Time PCR System, and the results were normalized to either $\beta$-actin or Gapdh expression.

RNA-Seq. After total RNA isolation as noted previously, RNA libraries were generated from 200 ng total RNA using the TruSeq RNA Sample Prep kit v2 (Illumina). The RNA integrity number was confirmed to be greater than 8.0 for all samples. Libraries were sequenced on the HiSeq4000 (Illumina), aligned to the mouse genome mm10 using TopHat (73), and assembled using StringTie (74). Differentially expressed genes were identified using edgeR (75) using default settings. Data were deposited in a public repository (GEO GSE179284).

Analytic methods. Blood glucose was measured using a Freestyle Lite glucometer (Abbott). Mouse insulin was measured using the Ultrasensitive Mouse Insulin ELISA (Alpco Diagnostics) as per the manufacturer's instructions. Human insulin was measured using the Human Insulin ELISA (Alpco Diagnostics). For blood gas and blood metabolite measurements, blood was drawn from mice using submandibular vein puncture and immediately transferred to the pHOx Ultra system (Nova Biomedical) according to the manufacturer's instructions.

Informatics. Publicly available human islet microarray data sets comparing the transcriptome of ND and T2DM islets were queried with GEO using the search terms: "T2DM/type 2 diabetes," "pancreatic islet/islet," and "Homo sapiens." From our search, we obtained 5 studies containing at least 5 ND and 5 T2DM donors from GEO: GSE38642 (28), GSE25724 (29), GSE20966 (30), GSE76894 (31), and GSE76895 (31). The raw data were downloaded from GEO's website. In studies where glycemia was assessed (GEO GSE38642; GSE78695), ND samples with impaired glucose tolerance (HbA1c $\geq 5.7 \%$ or noted), unknown glycemia, and type 3 diabetes were excluded. A total of 156 ND and 81 T2D samples were retained for downstream analysis. The raw $\mathrm{CEL}$ files were loaded into the R package Affy v1.68 for normalization of probe intensity by the MAS 5.0 algorithm (76). After normalization, low expressing probes ( $\log _{2}$ [intensity] $\leq 5$ ) were excluded if they were expressed in less than $50 \%$, minus 1 sample. BioMart was then used to annotate the probes to their corresponding target genes, and only probes that successfully annotated to coding genes were retained for downstream analysis. The filtered expression matrix was subsequently loaded into the LIMMA v3.46.0 (Linear Models for Microarray data) package, and differential analysis between ND and T2DM islets was performed for each data set using the empirical Bayes procedure (77). As previously discussed, there are many challenges in combining differential analysis for multiple probes mapping to one gene (78). We utilized the approach from Rhodes et al. and selected the probe with the smallest $P$ value for each gene (79). To combine the differential expression analysis from each of the 5 studies, we then inputted the filtered analysis from LIMMA into MetaVolcanoR v1.4.0 (80). We then per- formed random-effect modeling to assess and summarize the variance of expression for each gene between ND and T2DM islets. Single-cell RNASeq data from ND and T2DM human $\beta$ cell samples (GSE83139; ref. 17) were also obtained from the GEO repository. Pearson correlation analysis of islet gene expression from GEO GSE38642 was conducted using MATLAB 2015b (MathWorks). Ontological analyses of genes significantly correlated with SLC4A4 and genes found to be differentially expressed by RNA-Seq were conducted using Metascape (81) or WebGESTALT (82) against KEGG and protein analysis through evolutionary relationshipscurated (PANTHER-curated) pathways and were subsequently visualized using Cytoscape (83). GSEA for KEGG pathways was conducted using GSEA 4.0.1 with default settings (84). Principal component analysis of RNA-Seq transcript matrix was conducted in R3.6.1 using the ggfortify package with default settings (85).

Statistics. Statistical analysis performed for each data set is described within the associated figure legend. All statistical testing was performed using GraphPad Prism v.8.2 using either a 2-tailed Student's $t$ test with correction for multiple comparisons using the Holm-Sidak method or 1- or 2-way ANOVA with correction for multiple comparisons using Dunnett's or Tukey's method wherever appropriate and noted. Data are presented as mean \pm SEM. $P$ values of less than 0.05 were considered significant.

Study approval. All experimental procedures involving animals were approved by Mayo Clinic IACUC and performed in compliance with NIH policies on use of laboratory animals.

\section{Author contributions}

MB contributed to study design, conducted experiments, and assisted with the data analysis and interpretation and preparation of the manuscript. HH conducted experiments and assisted with the data analysis and interpretation and preparation of the manuscript. KR contributed to study design, conducted experiments, and reviewed the manuscript. NJ contributed to study design, conducted experiments, and reviewed the manuscript. TH contributed to conducted experiments and reviewed the manuscript. AS contributed to conducted experiments and reviewed the manuscript. SS contributed to conducted experiments and reviewed the manuscript. GES contributed to study design and preparation of the manuscript. YSP contributed to study design, data analysis and interpretation, and preparation of the manuscript. MFR contributed to study design, data analysis and interpretation, and preparation of the manuscript. AVM designed and interpreted the studies and wrote the manuscript. AVM is the guarantor of this work and, as such, had full access to all data in the study and takes responsibility for the integrity and accuracy of data analysis.

\section{Acknowledgments}

We acknowledge funding support from the NIH (R01DK098468 to AVM, R01DK128844 to AVM and MFR, and F99DK123834 to $\mathrm{MB}$ ) and the Center for Biomedical Discovery (Mayo Clinic). Schematics were generated using the premium service from BioRender.com.

Address correspondence to: Y.S. Prakash, Michael F. Romero, or Aleksey V. Matveyenko, 200 First Street SW, Rochester, Minnesota 55905, USA. Phone: 507.255.7481; Email: prakash.ys@mayo.edu (YSP). Phone: 507.254.8127; Email: romero.michael@mayo.edu (MRF). Phone:507.293.0843.Email: matveyenko.aleksey@mayo.edu (AVM). 
1. Rutter GA, et al. Pancreatic $\beta$-cell identity, glucose sensing and the control of insulin secretion. Biochem J. 2015;466(2):203-218.

2. Kahn SE, et al. The beta cell lesion in type 2 diabetes: there has to be a primary functional abnormality. Diabetologia. 2009;52(6):1003-1012.

3. Haythorne E, et al. Diabetes causes marked inhibition of mitochondrial metabolism in pancreatic $\beta$-cells. Nat Commun. 2019;10(1):2474.

4. Laybutt DR, et al. Genetic regulation of metabolic pathways in beta-cells disrupted by hyperglycemia. J Biol Chem. 2002;277(13):10912-10921.

5. Montemurro $\mathrm{C}$, et al. IAPP toxicity activates HIF1alpha/PFKFB3 signaling delaying beta-cell loss at the expense of beta-cell function. Nat Commun. 2019;10(1):2679.

6. Talchai $C$, et al. Pancreatic $\beta$ cell dedifferentiation as a mechanism of diabetic $\beta$ cell failure. Cell. 2012;150(6):1223-1234.

7. Bensellam M, et al. Mechanisms of $\beta$-cell dedifferentiation in diabetes: recent findings and future research directions. JEndocrinol. 2018;236(2):R109-R143.

8. Kim-Muller JY, et al. Aldehyde dehydrogenase 1a3 defines a subset of failing pancreatic $\beta$ cells in diabetic mice. Nat Commun. 2016;7:12631.

9. Webb BA, et al. Dysregulated pH: a perfect storm for cancer progression. Nat Rev Cancer. 2011;11(9):671-677.

10. Persi E, et al. Systems analysis of intracellular $\mathrm{pH}$ vulnerabilities for cancer therapy. Nat Commun. 2018;9(1):2997.

11. McBrian MA, et al. Histone acetylation regulates intracellular pH. Mol Cell. 2013;49(2):310-321.

12. Pouyssegur J, et al. Cytoplasmic pH, a key determinant of growth factor-induced DNA synthesis in quiescent fibroblasts. FEBS Lett. 1985;190(1):115-119.

13. Boedtkjer E, et al. Physiology, pharmacology and pathophysiology of the $\mathrm{pH}$ regulatory transport proteins NHE1 and NBCn1: similarities, differences, and implications for cancer therapy. Curr Pharm Des. 2012;18(10):1345-1371.

14. Boyer MJ, Tannock IF. Regulation of intracellular pH in tumor cell lines: influence of microenvironmental conditions. Cancer Res. 1992;52(16):4441-4447.

15. Pouyssegur J, et al. Hypoxia signalling in cancer and approaches to enforce tumour regression. Nature. 2006;441(7092):437-443.

16. Gorbatenko A, et al. Regulation and roles of bicarbonate transporters in cancer. Front Physiol. 2014;5:130.

17. Wang YJ, et al. Single-cell transcriptomics of the human endocrine pancreas. Diabetes. 2016;65(10):3028-3038.

18. Romero MF, Boron WF. Electrogenic Na+/HCO3 cotransporters: cloning and physiology. Annu Rev Physiol. 1999;61(1):699-723.

19. Romero MF, et al. The SLC4 family of bicarbonate $(\mathrm{HCO}(3)(-))$ transporters. Mol Aspects Med. 2013;34(2-3):159-182.

20. Romero MF, et al. Expression cloning and characterization of a renal electrogenic $\mathrm{Na}+/ \mathrm{HCO}_{3}-$ cotransporter. Nature. 1997;387(6631):409-413.

21. Gallagher FA, et al. Magnetic resonance imaging of $\mathrm{pH}$ in vivo using hyperpolarized $13 \mathrm{C}$-labelled bicarbonate. Nature. 2008;453(7197):940-943.
22. Parks SK, Pouyssegur J. The $\mathrm{Na}(+) / \mathrm{HCO} 3(-)$ co-transporter SLC4A4 plays a role in growth and migration of colon and breast cancer cells. JCell Physiol. 2015;230(8):1954-1963.

23. Bulur N, et al. Expression of the electrogenic $\mathrm{Na}+-\mathrm{HCO} 3-$-cotransporter NBCe1 in tumoral insulin-producing BRIN-BD11 cells. Cell Physiol Biochem. 2009;24(3-4):187-192.

24. Gunawardana SC, et al. Nutrient-stimulated insulin secretion in mouse islets is critically dependent on intracellular pH. BMC Endocr Disord. 2004;4(1):1.

25. Gunawardana SC, Sharp GW. Intracellular pH plays a critical role in glucose-induced time-dependent potentiation of insulin release in rat islets. Diabetes. 2002;51(1):105-113.

26. Gunawardana SC, et al. Amiloride derivatives enhance insulin release in pancreatic islets from diabetic mice. BMC Endocr Disord. 2005;5:9.

27. Gunawardana SC, et al. Dimethyl amiloride improves glucose homeostasis in mouse models of type 2 diabetes. Am J Physiol Endocrinol Metab. 2008;294(6):E1097-E1108.

28. Taneera J, et al. A systems genetics approach identifies genes and pathways for type 2 diabetes in human islets. Cell Metab. 2012;16(1):122-134.

29. Dominguez V, et al. Class II phosphoinositide 3-kinase regulates exocytosis of insulin granules in pancreatic beta cells. J Biol Chem 2011;286(6):4216-4225.

30. Marselli L, et al. Gene expression profiles of betacell enriched tissue obtained by laser capture microdissection from subjects with type 2 diabetes. PLoS One. 2010;5(7):e11499.

31. Solimena M, et al. Systems biology of the IMIDIA biobank from organ donors and pancreatectomised patients defines a novel transcriptomic signature of islets from individuals with type 2 diabetes. Diabetologia. 2018;61(3):641-657.

32. Adam J, et al. Fumarate hydratase deletion in pancreatic $\beta$ cells leads to progressive diabetes. Cell Rep. 2017;20(13):3135-3148.

33. Hunter CS, Stein RW. Evidence for loss in identity, de-differentiation, and trans-differentiation of islet $\beta$-cells in type 2 diabetes. Front Genet. 2017;8:35.

34. Cinti F, et al. Evidence of $\beta$-cell dedifferentiation in human type 2 diabetes. J Clin Endocrinol Metab. 2016;101(3):1044-1054.

35. Vairamani K, et al. $\mathrm{NBCe} 1 \mathrm{Na}^{+}-\mathrm{HCO}^{-}$cotransporter ablation causes reduced apoptosis following cardiac ischemia-reperfusion injury in vivo. World J Cardiol. 2018;10(9):97-109.

36. Theparambil SM, et al. Astrocytes regulate brain extracellular $\mathrm{pH}$ via a neuronal activity-dependent bicarbonate shuttle. Nat Commun . 2020;11(1):1-15.

37. Wicksteed B, et al. Conditional gene targeting in mouse pancreatic ß-Cells: analysis of ectopic Cre transgene expression in the brain. Diabetes. 2010;59(12):3090-3098.

38. Brouwers B, et al. Impaired islet function in commonly used transgenic mouse lines due to human growth hormone minigene expression. Cell Metab. 2014;20(6):979-990.

39. Winzell MS, Ahrén B. The high-fat diet-fed mouse: a model for studying mechanisms and treatment of impaired glucose tolerance and type 2 diabetes.
Diabetes. 2004;53(suppl 3):S215-S219.

40. Riant E, et al. Estrogens protect against high-fat diet-induced insulin resistance and glucose intolerance in mice. Endocrinology. 2009;150(5):2109-2117.

41. Gawenis LR, et al. Colonic anion secretory defects and metabolic acidosis in mice lacking the NBC1 Na+/HCO3- cotransporter. J Biol Chem. 2007;282(12):9042-9052.

42. Chen A, et al. Targeted NBCe1A deletion causes proximal RTA: whole nbce1 (sh_nbce1) KO vs. nbce1A KO mice. J Am Soc Nephrol. 2014;25:71A.

43. Kitamura YI, et al. FoxO1 protects against pancreatic beta cell failure through NeuroD and MafA induction. Cell Metab. 2005;2(3):153-163.

44. Ruminot I, et al. NBCe1 mediates the acute stimulation of astrocytic glycolysis by extracellular K+. J Neurosci. 2011;31(40):14264-14271.

45. Polonsky K, et al. Quantitative study of insulin secretion and clearance in normal and obese subjects. J Clin Invest. 1988;81(2):435-441.

46. Ashcroft FM, et al. Stimulus-secretion coupling in pancreatic $\beta$ cells. JCell Biochem. 1994;55(S199 4A):54-65.

47. Taylor BL, et al. Nkx6.1 is essential for maintaining the functional state of pancreatic beta cells. Cell Rep. 2013;4(6):1262-1275.

48. Pullen T, Rutter G. When less is more: the forbidden fruits of gene repression in the adult $\beta$-cell. Diabetes Obes Metab. 2013;15(6):503-512.

49. Pullen TJ, et al. Identification of genes selectively disallowed in the pancreatic islet. Islets. 2010;2(2):89-95.

50. Nomoto H, et al. Activation of the HIF1 $\alpha /$ PFKFB3 stress response pathway in beta cells in type 1 diabetes. Diabetologia. 2020;63(1):149-161.

51. Zehetner J, et al. PVHL is a regulator of glucose metabolism and insulin secretion in pancreatic beta cells. Genes Dev. 2008;22(22):3135-3146.

52. Puri S, et al. A role for von Hippel-Lindau protein in pancreatic beta-cell function. Diabetes. 2009;58(2):433-441.

53. Nguyen TT, Bonanno JA. Bicarbonate, NBCe1, NHE, and carbonic anhydrase activity enhance lactate- $\mathrm{H}+$ transport in bovine corneal endothelium. Invest Ophthalmol Vis Sci. 2011;52(11):8086-8093.

54. Petersen C, et al. MCT1 and MCT4 expression and lactate flux activity increase during white and brown adipogenesis and impact adipocyte metabolism. Sci Rep. 2017;7(1):1-13.

55. Stüwe L, et al.pH dependence of melanoma cell migration: protons extruded by NHE1 dominate protons of the bulk solution. J Physiol. 2007;585(2):351-360.

56 . Vierra NC, et al. Type 2 diabetes-associated $\mathrm{K}+$ channel TALK-1 modulates $\beta$-cell electrical excitability, second-phase insulin secretion, and glucose homeostasis. Diabetes. 2015;64(11):3818-3828.

57. Brun T, et al. Diabetogenic milieus induce specific changes in mitochondrial transcriptome and differentiation of human pancreatic islets. Hum Mol Genet. 2015;24(18):5270-5284.

58. Olsson AH, et al. Decreased expression of genes involved in oxidative phosphorylation in human pancreatic islets from patients with type 2 diabetes. Eur JEndocrinol. 2011;165(4):589-595.

59. van der Meulen T, et al. Virgin beta cells persist 
throughout life at a neogenic niche within pancreatic islets. Cell Metab. 2017;25(4):911-926.

60. Alvarez-Dominguez JR, et al. Circadian entrainment triggers maturation of human in vitro islets. Cell Stem Cell. 2020;26(1):108-122.

61. Rosado-Olivieri EA, et al. YAP inhibition enhances the differentiation of functional stem cell-derived insulin-producing $\beta$ cells. Nat Commun. 2019;10(1):1464.

62. Dhawan S, et al. DNA methylation directs functional maturation of pancreatic $\beta$ cells. J Clin Invest. 2015;125(7):2851-2860.

63. Southgate CD, et al. Targeted disruption of the murine erythroid band 3 gene results in spherocytosis and severe haemolytic anaemia despite a normal membrane skeleton. Nat Genet. 1996;14(2):227-230.

64. Stiernet P, et al. Glucose-induced cytosolic pH changes in beta-cells and insulin secretion are not causally related: studies in islets lacking the $\mathrm{Na}+/ \mathrm{H}+$ exchangeR NHE1.J Biol Chem. 2007;282(34):24538-24546.

65. Shepherd RM, Henquin J-C. The role of metabolism, cytoplasmic $\mathrm{Ca} 2+$, and $\mathrm{pH}$-regulating exchangers in glucose-induced rise of cytoplasmic $\mathrm{pH}$ in normal mouse pancreatic islets. J Biol Chem. 1995;270(14):7915-7921.

66. Knudsen JG, et al. Dysregulation of glucagon secretion by hyperglycemia-induced sodiumdependent reduction of ATP production. Cell Metab. 2019;29(2):430-442.

67. Khandoudi N, et al. Inhibition of the cardiac electrogenic sodium bicarbonate cotransporter reduces ischemic injury. Cardiovasc Res. 2001;52(3):387-396.
68. Nordstrom T, et al. Tannic acid inhibits electrogenic $\mathrm{Na}+\mathrm{HCO} 3-$ co-transporter activity in embryonic neural stem cell-derived radial glial-like cells. Neuroreport. 2020;31(1):57-63.

69. Ch'en FF, et al. S0859, an N-cyanosulphonamide inhibitor of sodium-bicarbonate cotransport in the heart. Br J Pharmacol. 2008;153(5):972-982.

70. Tiano JP, et al. Effect of targeted estrogen delivery using glucagon-like peptide- 1 on insulin secretion, insulin sensitivity and glucose homeostasis. Sci Rep. 2015;5:10211.

71. Bevensee $\mathrm{MO}$, et al. An electrogenic $\mathrm{Na}(+)$ $\mathrm{HCO}(-)$ (3) cotransporter (NBC) with a novel $\mathrm{COOH}$-terminus, cloned from rat brain. $\mathrm{Am} \mathrm{J}$ Physiol Cell Physiol. 2000;278(6):C1200-C1211.

72. Rakshit K, et al. Bmal1 is required for beta cell compensatory expansion, survival and metabolic adaptation to diet-induced obesity in mice. Diabetologia. 2016;59(4):734-743.

73. Trapnell C, et al. Differential gene and transcript expression analysis of RNA-seq experiments with TopHat and Cufflinks. Nat Protoc. 2012;7(3):562-578.

74. Pertea M, et al. StringTie enables improved reconstruction of a transcriptome from RNA-seq reads. Nat Biotechnol. 2015;33(3):290-295.

75. Robinson MD, et al. edgeR: a Bioconductor package for differential expression analysis of digital gene expression data. Bioinformatics. 2010;26(1):139-140.

76. Gautier L, et al. affy-analysis of Affymetrix GeneChip data at the probe level. Bioinformatics. 2004;20(3):307-315.

77. Ritchie ME, et al. limma powers differential expression analyses for RNA-sequencing and microarray studies. Nucleic Acids Res. 2015;43(7):e47.

78. Ramasamy A, et al. Key issues in conducting a meta-analysis of gene expression microarray datasets. PLoS Med. 2008;5(9):e184.

79. Rhodes DR, et al. Meta-analysis of microarrays: interstudy validation of gene expression profiles reveals pathway dysregulation in prostate cancer. Cancer Res. 2002;62(15):4427-4433.

80. Prada C, et al. MetaVolcanoR: Gene Expression Meta-analysis Visualization Tool. Version 1.4.0. Updated 2020. Accessed July 26, 2021. https:// rdrr.io/bioc/MetaVolcanoR/.

81. Zhou Y, et al. Metascape provides a biologist-oriented resource for the analysis of systems-level datasets. Nat Commun. 2019;10(1):1-10.

82. Liao Y, et al. WebGestalt 2019: gene set analysis toolkit with revamped UIs and APIs. Nucleic Acids Res. 2019;47(W1):W199-W205.

83. Shannon P, et al. Cytoscape: a software environment for integrated models of biomolecular interaction networks. Genome Res. 2003;13(11):2498-2504.

84. Subramanian A, et al. Gene set enrichment analysis: a knowledge-based approach for interpreting genome-wide expression profiles. Proc Natl Acad Sci US A. 2005;102(43):15545-15550.

85. Tang Y, et al. ggfortify: unified interface to visualize statistical results of popular R packages. $R J$. 2016;8(2):474-485.

86. Her TK, et al. Dietary carbohydrates modulate metabolic and $\beta$-cell adaptation to high-fat diet-induced obesity. Am J Physiol Endocrinol Metab. 2020;318(6):E856-E865. 